Homology, Homotopy and Applications, vol.4(2), 2002, pp.141-163

\title{
NONSPLIT EXTENSIONS OF MODULAR LIE ALGEBRAS OF RANK 2
}

\author{
A.S. DZHUMADIL'DAEV AND S.S. IBRAEV
}

(communicated by Larry Lambe)

\begin{abstract}
Second cohomology groups of irreducible representations of classical Lie algebras $A_{2}, B_{2}$ and $G_{2}$ over an algebraically closed field of characteristic $p>h$ are calculated. Here $h$ is the Coxeter number.
\end{abstract}

To Jan-Erik Roos on his sixty-fifth birthday

\section{Formulation of the main result}

Levi-Mal'cev theorem has cohomological origin. It states that any finite-dimensional extension of a finite-dimensional simple Lie algebra over a field of characteristic 0 is split. In case of characteristic $p>0$ any Lie algebra has at least one nonsplit extension and the number of irreducible modules with such a property is finite [8]. For example, the 3 -dimensional simple Lie algebra $A_{1}=s l_{2}$ has exactly one irreducible module, namely the $(p-1)$-dimensional module $V_{p-2}$, with $H^{2}\left(A_{1}, V_{p-2}\right) \neq 0[\mathbf{7}]$.

The aim of our paper is to calculate second cohomology groups with coefficients in an irreducible module for simple Lie algebras of rank $2: \mathfrak{g}=A_{2}, B_{2}$ and $G_{2}$. The field $\mathcal{K}$ is algebraically closed and has characteristic $p>h$, where $h$ is the Coxeter number. An irreducible $\mathfrak{g}$-module $V$ is called 2-peculiar, if $H^{2}(\mathfrak{g}, V) \neq 0$. Let $\kappa_{2}(\mathfrak{g})$ be the number of peculiar modules. From our results it follows that $\kappa_{2}(\mathfrak{g})=2,3,3$, for $\mathfrak{g}=A_{2}, B_{2}, G_{2}$ respectively. Let $L(\lambda)$ be an irreducible module with highest weight $\lambda$.

The main result of this paper is the following

Theorem 1.1. Let $\mathfrak{g}=A_{2}, B_{2}, G_{2}, p>h$ and $V$ be an irreducible $\mathfrak{g}$-module. Then $H^{2}(\mathfrak{g}, V)$ is trivial except in the following cases:

(a) $\mathfrak{g}=A_{2}, H^{2}\left(\mathfrak{g}, L\left((p-3) \lambda_{i}\right)\right) \cong L\left(\lambda_{i}\right)^{(1)}, \quad i=1,2$;

(b) $\mathfrak{g}=B_{2}, H^{2}\left(\mathfrak{g}, L\left((p-3) \lambda_{1}+2 \lambda_{2}\right)\right) \cong L\left(\lambda_{1}\right)^{(1)}$,

$$
\begin{gathered}
H^{2}\left(\mathfrak{g}, L\left(\lambda_{1}+(p-4) \lambda_{2}\right)\right) \cong L\left(\lambda_{2}\right)^{(1)}, \\
H^{2}\left(\mathfrak{g}, L\left((p-2)\left(\lambda_{1}+\lambda_{2}\right)\right)\right) \cong L\left(\lambda_{2}\right)^{(1)} ;
\end{gathered}
$$

Received February 16, 2001, revised February 8, 2002; published on July 12, 2002.

2000 Mathematics Subject Classification: 17B50, 17B56.

Key words and phrases: modular Lie algebras, nonsplit extensions, Levi-Mal'cev theorem, second cohomology groups, simple Lie algebras, restricted cohomology groups.

(c) 2002, A.S. Dzhumadil'daev and S.S. Ibraev. Permission to copy for private use granted. 


$$
\begin{aligned}
(c) \mathfrak{g}=G_{2}, H^{2}(\mathfrak{g}, L & \left.\left((p-5) \lambda_{1}+2 \lambda_{2}\right)\right) \cong L\left(\lambda_{1}\right)^{(1)}, \\
& H^{2}\left(\mathfrak{g}, L\left(4 \lambda_{1}+(p-3) \lambda_{2}\right)\right) \cong L\left(\lambda_{2}\right)^{(1)}, \\
& \left.H^{2}\left(\mathfrak{g}, L\left(3 \lambda_{1}+(p-2) \lambda_{2}\right)\right)\right) \cong L(0)^{(1)} .
\end{aligned}
$$

Here we use notations from $[\mathbf{1 1}]$. Let $\mathfrak{g}$ be a classical Lie algebra over the field $\mathcal{K}$, $G$ an algebraic group of the Lie algebra $\mathfrak{g}$. Recall that the Frobenius map is defined as the morphism $G \rightarrow G$ of the $\mathcal{K}$-group functor $G$ induced by the map $x \mapsto x^{p}$ on the function algebra $\mathcal{K}[G][\mathbf{1 1}]$. The normal subgroup $G_{1}$ is the scheme-theoretic kernel of this map. Let $T$ be the maximal torus on $G, X(T)$ be the character group of $T, R \subset X(T)$ be the root system and $R^{+}$be the set of positive roots on $R$. The simple roots $\alpha_{1}, \alpha_{2}, \ldots, \alpha_{n}$ corresponds to the Bourbaki table [3]. Let $\lambda_{1}, \lambda_{2}, \cdots, \lambda_{n}$ be the fundamental weights, $X(T)_{+}$be the set of dominant weights, $X_{1}(T)$ be the set of restricted dominant weights, i.e., $X_{1}(T)=\left\{\lambda=\sum_{i=1}^{n} r_{i} \lambda_{i} \in X(T): r_{i} \in\right.$ $\mathbb{Z}, 0 \leqslant r_{i}<p$, for all $\left.i\right\}$. Endow $X(T)$ by the usual order $: \lambda \leqslant \mu$ if and only if, there exist integers $r_{i} \geqslant 0$ such that $\mu-\lambda=\sum_{i=1}^{n} r_{i} \alpha_{i}$. For any $T$-module $V$ and any $\mu \in X(T)$ denote by $V_{\mu}$ its weight subspace in $V$.

There exists an algebra $\mathfrak{g}_{\mathbb{Z}}$ over $\mathbb{Z}$ such that $\mathfrak{g}_{\mathbb{Z}} \otimes \mathcal{K} \cong \mathfrak{g}$. In $\mathfrak{g}_{\mathbb{Z}}$ one can choose a Chevalley basis, that coincides with a basis of the semi-simple complex Lie algebra. To any root $\alpha$ there corresponds a basic vector $e_{\alpha}$ of the Lie algebra $\mathfrak{g}_{\mathbb{Z}}$. If $\alpha, \beta \in R$, then $\left[e_{\alpha}, e_{\beta}\right]=N_{\alpha, \beta} e_{\alpha+\beta}$ for some integer $N_{\alpha, \beta}$. Identify $e_{\alpha}$ with $e_{\alpha} \otimes 1$. Note that the $p$-map $e \mapsto e^{[p]}$, defined on $\mathfrak{g}$, has the property $e_{\alpha}^{[p]}=0$ for any $\alpha \in R$.

Recall the definition of a Weyl module. Let $\mathfrak{g}_{\mathbb{C}}$ be a Lie algebra over the field of complex numbers $\mathbb{C}$. Consider an irreducible $\mathfrak{g}_{\mathbb{C}}$-module $V(\lambda)_{\mathbb{C}}$ with highest weight $\lambda$. It is known that there exists a $\mathbb{Z}$-submodule $V(\lambda)_{\mathbb{Z}}$ of the $\mathfrak{g}_{\mathbb{C}}$-module $V(\lambda)_{\mathbb{C}}$. Then $V(\lambda)=V(\lambda)_{\mathbb{Z}} \otimes \mathcal{K}$ is a $\mathfrak{g}$-module. The obtained module is called a Weyl module.

Let $B$ be the Borel subgroup of $G$ corresponding to the negative roots, $U$ be the unipotent radical of $B$ and $\mathfrak{u}$ be the Lie algebra of $U$. The Lie algebra $\mathfrak{u}$ is a nilpotent subalgebra of the Lie algebra $\mathfrak{g}$ and it spans basic vectors $e_{-\alpha}, \alpha \in R^{+}$. The Cartan subalgebra $\mathfrak{h}$ of the Lie algebra $\mathfrak{g}$ is a Lie algebra of the maximal torus $T$ of $G$. For any $\lambda \in X(T)$ one can define a one-dimensional module $\mathcal{K}_{\lambda}$ over $B$ using the isomorphism $B / U \cong T$. The induced $G$-module $H^{0}(\lambda)=I n d_{B}^{G} \mathcal{K}_{\lambda}$ is non-zero if and only if $\lambda \in X(T)_{+}$. If so, the socle $L(\lambda)$ of the induced module $H^{0}(\lambda)$ is a simple $G$-module with highest weight $\lambda$. It can also be constructed as the unique irreducible factor of the Weyl module $V(\lambda)$.

If $\lambda \in X_{1}(T)$, then $L(\lambda)$ remains simple as a $G_{1}$-module. Any simple $G_{1}$-module is defined uniquely by the highest weight $\lambda \in X_{1}(T)$ and it is isomorphic to $L(\lambda)$. The theory of restricted representations of the restricted Lie algebra $\mathfrak{g}$ is equivalent to the theory of representations of the group $G_{1}$.

A composition of a representation of $G$ in a vector space $V$ with the Frobenius map gives us a new representation with trivial action of $G_{1}$. Denote the obtained module by $V^{(1)}$. Thus this module as a module over the Lie algebra $\mathfrak{g}$ is a module with a trivial action. To any weight $\mu \in X(T)$ of the space $\mathrm{V}$ there corresponds the weight $p \mu$ of the space $V^{(1)}$. On the other hand, if $V_{1}$ is a $G$-module with trivial action of $G_{1}$ (or $\mathfrak{g}$ ) then there exists a unique $G$-module $V$, such that $V_{1}=V^{(1)}$. 
Denote this $G$-module $V$ by $V^{(-1)}$. For example, if $L$ is a $G$-module, then any cohomology group $H^{i}\left(G_{1}, L\right)$ is a $G$-module with trivial action of $G_{1}$ (or $\mathfrak{g}$ ). Therefore the module $H^{i}\left(G_{1}, L\right)^{(-1)}$ is a $G$-module with the above mentioned property.

Second cohomology groups of the adjoint representation of the Lie algebra $B_{2}$ in characteristic 3 was studied in $[\mathbf{1 3}],[\mathbf{6}]$. In [16], [11] first cohomology groups of modular Lie algebras with coefficients in irreducible modules are calculated. In [16] the non-triviality of first cohomology groups with coefficients in irreducible restricted modules with highest weights $p \lambda_{i}-\alpha_{i}, i=1,2, \ldots, n$, are proved. Here $\alpha_{i}, \lambda_{i}, \quad i=1,2, \ldots, n$ are the simple roots and fundamental weights. In [17] a connection between first cohomology groups of irreducible modules and second cohomology groups of restricted Weyl modules are studied.

\section{Connection between ordinary and restricted second coho- mology groups}

Consider the algebra $\mathfrak{g}$ as a restricted Lie algebra with the $p$-map $e \mapsto e^{[p]}, e \in \mathfrak{g}$. Let $U(\mathfrak{g})$ be the universal enveloping algebra of $\mathfrak{g}$ and $U(\mathfrak{g})^{+}$be a two sided ideal in $U(\mathfrak{g})$ such that $U(\mathfrak{g})$ is a direct sum of $\mathcal{K}$ and $U(\mathfrak{g})^{+}$. Let $P(\mathfrak{g})$ be the ideal generated by the elements $e^{p}-e^{[p]}, e \in \mathfrak{g}$. The factor-algebra $U_{0}(\mathfrak{g})=U(\mathfrak{g}) / P(\mathfrak{g})$ is called the restricted universal enveloping algebra of $\mathfrak{g}$.

Restricted cohomology groups of restricted Lie algebras were introduced by G.Hochschild in ([9]). The cohomology groups $H^{i}\left(G_{1}, V\right)$ for a $G_{1}$-module $V$ are equivalent to the restricted cohomology of the corresponding $\mathfrak{g}$-module $([\mathbf{1 1}]$, I.9, p.145 ). Let $H_{*}^{i}(\mathfrak{g}, V)$ denote the $i$-th restricted cohomology group of the restricted Lie algebra $\mathfrak{g}$ with coefficients in a restricted $\mathfrak{g}$-module $V$. By definition $H_{*}^{i}(\mathfrak{g}, V)=$ $\operatorname{Ext}_{U_{0}(\mathfrak{g})}^{i}(\mathcal{K}, V)$.

The projection $U(\mathfrak{g}) \rightarrow \mathcal{K}$ induces the projection $U_{0}(\mathfrak{g}) \rightarrow \mathcal{K}$. Denote its kernel by $U_{0}(\mathfrak{g})^{+}$. Then $U_{0}(\mathfrak{g})^{+}$is the image of $U(\mathfrak{g})^{+}$in $U_{0}(\mathfrak{g})$ of the canonical map $U(\mathfrak{g}) \rightarrow U_{0}(\mathfrak{g})$. A map of the corresponding cochain complexes is induced by the homomorphism $\psi \mapsto \psi^{0}$, where $\psi^{0}\left(s_{1}, s_{2}, \ldots, s_{i}\right)=\psi\left(s_{1}^{\prime}, s_{2}^{\prime}, \ldots, s_{i}^{\prime}\right), s_{j} \in U(\mathfrak{g})^{+}$ and $s_{j}^{\prime}$ are the canonical images in $U_{0}(\mathfrak{g})^{+}$.

Let now $C(V)$ be the cochain complex for the universal enveloping algebra $U(\mathfrak{g})$ of the Lie algebra $\mathfrak{g}$ with coefficients in the $\mathfrak{g}$-module $V$.

Let $C^{0}(V)$ stand for the subcomplex consisting of the cochains of the form $\psi^{0}$, where $\psi$ is a cochain for $U_{0}(\mathfrak{g})^{+}$with coefficients in $V$. Then we have an exact sequence of cochain complexes

$$
0 \rightarrow C^{0}(V) \rightarrow C(V) \rightarrow C(V) / C^{0}(V) \rightarrow 0 .
$$

Since the map $\psi \mapsto \psi^{0}$ is an isomorphism, we may identify $H^{i}\left(C^{0}(V)\right)$ with $H_{*}^{i}(\mathfrak{g}, V)$. This gives us the following exact sequence:

$$
\cdots \rightarrow H_{*}^{i}(\mathfrak{g}, V) \rightarrow H^{i}(\mathfrak{g}, V) \rightarrow H^{i}\left(C(V) / C^{0}(V)\right) \rightarrow H_{*}^{i+1}(\mathfrak{g}, V) \rightarrow \cdots
$$

In [9] Hochschild shows that for $i=1,2$

$$
H^{i}\left(C(V) / C^{0}(V)\right) \cong S\left(\mathfrak{g}, H^{i-1}(\mathfrak{g}, V)\right),
$$


where $S\left(\mathfrak{g}, H^{i-1}(\mathfrak{g}, V)\right)$ is the space of $p$-semilinear maps $\mathfrak{g} \rightarrow H^{i-1}(\mathfrak{g}, V)$. In [11] it was proved that for $i=1,2$ there is an isomorphism of $G$-modules

$$
S\left(\mathfrak{g}, H^{i-1}(\mathfrak{g}, V)\right) \cong H^{i-1}(\mathfrak{g}, V) \otimes \mathfrak{g}^{*}
$$

(proposition 9.20 , p. 160). It is evident that $H^{0}\left(C(V) / C^{0}(V)\right)=0$. The identification of $H_{*}^{i}(\mathfrak{g}, V)$ with $H^{i}\left(G_{1}, V\right)$ gives us the following exact sequence of $G$-modules:

$$
\begin{gathered}
0 \rightarrow H^{1}\left(G_{1}, V\right) \rightarrow H^{1}(\mathfrak{g}, V) \rightarrow H^{0}(\mathfrak{g}, V) \otimes \mathfrak{g}^{*} \rightarrow H^{2}\left(G_{1}, V\right) \rightarrow \\
\rightarrow H^{2}(\mathfrak{g}, V) \rightarrow H^{1}(\mathfrak{g}, V) \otimes \mathfrak{g}^{*} \rightarrow H^{3}\left(G_{1}, V\right) .
\end{gathered}
$$

Lemma 2.1. Let $V$ be a nontrivial irreducible $\mathfrak{g}$-module and $H^{1}(\mathfrak{g}, V)=0$. Then $H^{2}(\mathfrak{g}, V) \cong H^{2}\left(G_{1}, V\right)$ as $G$-modules.

Proof. Since $V$ is a nontrivial irreducible $\mathfrak{g}$-module, $H^{0}(\mathfrak{g}, V)=0$. The isomorphism follows from the exact sequence (1).

\section{Peculiar irreducible modules}

Call an irreducible $\mathfrak{g}$-module $V$ peculiar, if $H^{*}(\mathfrak{g}, V) \neq 0$. Let $\mathfrak{g}$ be a simple classical Lie algebra, $p>0, U_{0}(\mathfrak{g})$ its restricted universal enveloping algebra, $Z_{0}(\mathfrak{g})$ be the center of $U_{0}(\mathfrak{g})$. The central character $c_{V}: Z(\mathfrak{g}) \rightarrow \mathcal{K}$, maps each element $C \in Z_{0}(\mathfrak{g})$ to its unique eigenvalue $c_{V}(C)$ on $V$.

Let $\lambda, \mu \in X(T)$. We will say, that $\lambda$ and $\mu$ are connected, if $\lambda=w(\mu+\rho)-\rho$ for some $w \in W$. If $\lambda$ and $\mu$ are connected, then according to the linkage principal, $L(\mu)$ is a composition factor of Weyl module $V(\lambda)([\mathbf{1}]$, Corollary 3 of theorem 1$)$. This means that the maximal submodule of Weyl module $V(\lambda)$ is generated by highest vectors with weights connected with $\lambda$. If $M(\lambda)$ is a maximal submodule of the Weyl module $V(\lambda)$, then the following sequence is exact

$$
0 \rightarrow M(\lambda) \rightarrow V(\lambda) \rightarrow V(\lambda) / M(\lambda) \rightarrow 0 .
$$

The corresponding long exact cohomological sequence shows that the highest weights of peculiar modules are connected.

Lemma 3.1. Let $L(\lambda)$ be a peculiar module. Then $\lambda \in X_{1}(T)$ and $\lambda=w(\rho)-\rho+p \nu$ where $\nu \in X(T), w \in W$.

Proof. According to ([10], theorem 2.1) two modules with connected highest weights have just the same central characters. The trivial module is peculiar. It is evident that the central character of the trivial module is equal to zero. According to the linkage principle highest weights of peculiar modules are connected with the highest weight 0 . It is known that cohomologies of non-restricted modules are trivial $([\boldsymbol{7}])$. Thus, the highest weight of a peculiar module has the form $\lambda=w(\rho)-\rho+p \nu \in X_{1}(T)$, where $\nu \in X(T)$ and $w$ runs through elements of Weyl group $W$. 
Corollary 3.2. The lists of possible highest weights of any peculiar module of a simple classical Lie algebra $\mathfrak{g}$ of rank two are given below

$$
\begin{gathered}
\mathfrak{g}=A_{2} \\
0,(p-2) \lambda_{1}+\lambda_{2}, \lambda_{1}+(p-2) \lambda_{2},(p-3) \lambda_{1},(p-3) \lambda_{2},(p-2)\left(\lambda_{1}+\lambda_{2}\right) ; \\
\mathfrak{g}=B_{2} \\
0,(p-2) \lambda_{1}+2 \lambda_{2}, \lambda_{1}+(p-2) \lambda_{2},(p-3) \lambda_{1}+2 \lambda_{2}, \\
\lambda_{1}+(p-4) \lambda_{2},(p-3) \lambda_{1},(p-4) \lambda_{2},(p-2)\left(\lambda_{1}+\lambda_{2}\right) ; \\
\mathfrak{g}=G_{2} \\
0,(p-2) \lambda_{1}+\lambda_{2}, 3 \lambda_{1}+(p-2) \lambda_{2},(p-5) \lambda_{1}+2 \lambda_{2}, 4 \lambda_{1}+(p-3) \lambda_{2}, \\
(p-6) \lambda_{1}+2 \lambda_{2}, 4 \lambda_{1}+(p-4) \lambda_{2},(p-6) \lambda_{1}+\lambda_{2}, \\
3 \lambda_{1}+(p-4) \lambda_{2},(p-5) \lambda_{1},(p-3) \lambda_{2},(p-2)\left(\lambda_{1}+\lambda_{2}\right) .
\end{gathered}
$$

Proof. We show detailed calculations only in the case of $A_{2}$. For other algebras the calculations are similar. So, let $\mathfrak{g}=A_{2}$. The Weyl group has 6 elements $1, s_{1}, s_{2}, s_{1} s_{2}, s_{2} s_{1}, s_{1} s_{2} s_{1}$. Here $s_{i}$ corresponds to $s_{i}(\mu)=\mu-\frac{2\left(\mu, \alpha_{i}\right)}{\left(\alpha_{i}, \alpha_{i}\right)} \alpha_{i}$. The halfsum of positive roots is equal to $\rho=\alpha_{1}+\alpha_{2}$. It is evident that, to the neutral element 1 corresponds a peculiar highest weight $\lambda=0$. For $s_{1}$ we have

$$
\begin{gathered}
\lambda=s_{1}(\rho)-\rho+p \nu= \\
s_{1}\left(\alpha_{1}+\alpha_{2}\right)-\alpha_{1}-\alpha_{2}+p \nu=-\alpha_{1}+p \nu=-2 \lambda_{1}+\lambda_{2}+p \nu .
\end{gathered}
$$

Since $\lambda$ is restricted and dominant, $\nu=\lambda_{1}$. So $\lambda=s_{1}(\rho)-\rho+p \nu=(p-2) \lambda_{1}+\lambda_{2}$ may be a peculiar weight corresponding to $s_{1}$. Similarly,

$$
\begin{array}{r}
\lambda=s_{2}(\rho)-\rho+p \nu=-\alpha_{2}+p \nu=\lambda_{1}-2 \lambda_{2}+p \nu=\lambda_{1}+(p-2) \lambda_{2}, \\
\lambda=s_{1} s_{2}(\rho)-\rho+p \nu=-2 \alpha_{1}-\alpha_{2}+p \nu=-3 \lambda_{1}+p \nu=(p-3) \lambda_{1}, \\
\lambda=s_{2} s_{1}(\rho)-\rho+p \nu=-\alpha_{1}-2 \alpha_{2}+p \nu=-3 \lambda_{2}+p \nu=(p-3) \lambda_{2}, \\
\lambda=s_{1} s_{2} s_{1}(\rho)-\rho+p \nu=-2 \alpha_{1}-2 \alpha_{2}+p \nu=-2 \lambda_{1}-2 \lambda_{2}+p \nu= \\
(p-2)\left(\lambda_{1}+\lambda_{2}\right) .
\end{array}
$$

Lemma 3.3. Let $\mathfrak{g}=A_{2}$. Then as $G$-modules,

$$
\begin{array}{r}
H^{0}(0)=L(0), H^{0}\left((p-3) \lambda_{1}\right)=L\left((p-3) \lambda_{1}\right), H^{0}\left((p-3) \lambda_{2}\right)= \\
L\left((p-3) \lambda_{2}\right) ;
\end{array}
$$




$$
\begin{gathered}
H^{0}\left((p-2) \lambda_{1}+\lambda_{2}\right) / L\left((p-2) \lambda_{1}+\lambda_{2}\right) \cong L\left((p-3) \lambda_{1}\right) \\
H^{0}\left(\lambda_{1}+(p-2) \lambda_{2}\right) / L\left(\lambda_{1}+(p-2) \lambda_{2}\right) \cong L\left((p-3) \lambda_{2}\right) \\
H^{0}\left((p-2)\left(\lambda_{1}+\lambda_{2}\right)\right) / L\left((p-2)\left(\lambda_{1}+\lambda_{2}\right)\right) \cong L(0) .
\end{gathered}
$$

Proof. See $[\mathbf{4}],[\mathbf{1 4}],[\mathbf{1 2}]$.

Lemma 3.4. Let $\mathfrak{g}=B_{2}$ and $p>3$. Then

$$
\begin{gathered}
H^{0}(0)=L(0), H^{0}\left((p-3) \lambda_{1}\right)=L\left((p-3) \lambda_{1}\right), H^{0}\left((p-4) \lambda_{2}\right)= \\
L\left((p-4) \lambda_{2}\right) ; \\
H^{0}\left((p-2) \lambda_{1}+2 \lambda_{2}\right) / L\left((p-2) \lambda_{1}+2 \lambda_{2}\right) \cong L\left((p-3) \lambda_{1}+2 \lambda_{2}\right) ; \\
H^{0}\left(\lambda_{1}+(p-2) \lambda_{2}\right) / L\left(\lambda_{1}+(p-2) \lambda_{2}\right) \cong L\left(\lambda_{1}+(p-4) \lambda_{2}\right) ; \\
H^{0}\left((p-3) \lambda_{1}+2 \lambda_{2}\right) / L\left((p-3) \lambda_{1}+2 \lambda_{2}\right) \cong L\left((p-3) \lambda_{1}\right) ; \\
H^{0}\left(\lambda_{1}+(p-4) \lambda_{2}\right) / L\left(\lambda_{1}+(p-4) \lambda_{2}\right) \cong L\left((p-4) \lambda_{2}\right) ; \\
H^{0}\left((p-2)\left(\lambda_{1}+\lambda_{2}\right)\right) / L\left((p-2)\left(\lambda_{1}+\lambda_{2}\right)\right) \cong L\left(\lambda_{1}+(p-2) \lambda_{1}\right) .
\end{gathered}
$$

Proof. Recall that the element of maximal length of the Weyl group is $w_{0}=-1$ for $\mathfrak{g}=B_{2}$. Since in this case, $V(\lambda)=H^{0}\left(-w_{0}(\lambda)\right)^{*}=H^{0}(\lambda)^{*}$, the maximal submodule of the Weyl module is isomorphic to the factor-module $H^{0}(\lambda) / L(\lambda)$. Therefore it is enough to prove that for any of the considered modules $H^{0}(\lambda)$, the maximal submodule of the corresponding Weyl module $V(\lambda)$ coincides with an irreducible module mentioned in the lemma.

Let $\left\{e_{1}, e_{2}, e_{3}, e_{4}, h_{1}, h_{2}, f_{1}, f_{2}, f_{3}, f_{4}\right\}$ be the Chevalley basis of the Lie algebra $\mathfrak{g}$. Vectors in the module $V(\lambda)$ can be presented as linear combinations of monomials like

$$
v_{i, j, k, s}:=\frac{f_{4}^{s} f_{1}^{k} f_{3}^{j} f_{2}^{i}}{s ! k ! j ! i !} \otimes v_{\lambda},
$$

where $v_{\lambda}$ is the highest vector and $\left\{f_{1}, f_{2}, f_{3}, f_{4}\right\}$ is the basis of $\mathfrak{u}$. The actions of the elements $e_{1}, e_{2}$ on the monomials $v_{i, j, k, s}$ are defined by

$$
\begin{gathered}
e_{1} v_{i, j, k, s}=(s+1) v_{i, j-2, k, s+1}-(i+1) v_{i+1, j-1, k, s}+\left(m_{1}+1+i-j-k\right) v_{i, j, k-1, s}, \\
e_{2} v_{i, j, k, s}=2(k+1) v_{i, j-1, k+1, s}-(j+1) v_{i, j+1, k, s-1}+\left(m_{2}+1-i\right) v_{i-1, j, k, s},
\end{gathered}
$$

where $\lambda=m_{1} \lambda_{1}+m_{2} \lambda_{2}$.

Let

$$
v_{i, k}^{m_{1}, m_{2}}=\sum_{0 \leqslant j+s \leqslant k, 0 \leqslant j+2 s \leqslant i} a_{j, s} v_{i-j-2 s, j, k-j-s, s}
$$

be the vector in the space $V(\lambda)$ with weight $\lambda-k \alpha_{1}-i \alpha_{2}$. Call it normal, if $a_{0,0} \neq 0$. It is known that highest vectors of proper submodules of $V(\lambda)$ are normal $([\mathbf{1 5}])$.

It is evident that normal vectors of the modules $V\left((p-3) \lambda_{1}\right), V\left((p-4) \lambda_{2}\right)$ cannot serve as highest vectors. So, the modules $V\left((p-3) \lambda_{1}\right), V\left((p-4) \lambda_{2}\right)$ have no proper 
submodules. Therefore, they are irreducible and are equal to the corresponding induced modules.

Suppose now that $\lambda=(p-3) \lambda_{1}+2 \lambda_{2}$. Then highest vectors can be found among the normal vectors $v_{i, k}^{p-3,2}, i \leqslant 2, k \leqslant p-3$.

We now show that $v_{1,1}^{p-3,2}, v_{2,2}^{p-3,2}$ cannot serve as a highest vector. Suppose that

$$
\begin{gathered}
v_{1,1}^{p-3,2}=a_{1} v_{1,0,1,0}+b_{1} v_{0,1,0,0} \\
v_{2,2}^{p-3,2}=a_{2} v_{2,0,2,0}+b_{2} v_{1,1,1,0}+c_{2} v_{2,0,2,0}+d_{2} v_{1,1,1,0}
\end{gathered}
$$

are highest vectors. Then

$$
\begin{gathered}
e_{1} v_{1,1}^{p-3,2}=e_{1}\left(a_{1} v_{1,0,1,0}+b_{1} v_{0,1,0,0}\right)=0 \Rightarrow-2 a_{1}-b_{1}=0, \\
e_{2} v_{1,1}^{p-3,2}=e_{2}\left(a_{1} v_{1,0,1,0}+b_{1} v_{0,1,0,0}\right)=0 \Rightarrow \\
2 a_{1}+2 b_{1}=0 ; \\
e_{1} v_{2,2}^{p-3,2}=e_{1}\left(a_{2} v_{2,0,2,0}+b_{2} v_{1,1,1,0}+c_{2} v_{2,0,2,0}+d_{2} v_{1,1,1,0}\right)=0 \Rightarrow \\
c_{2}=b_{2}=a_{2}=0 ; \\
e_{2} v_{2,2}^{p-3,2}=e_{2}\left(a_{2} v_{2,0,2,0}+b_{2} v_{1,1,1,0}+c_{2} v_{2,0,2,0}+d_{2} v_{1,1,1,0}\right)=0 \Rightarrow \\
a_{2}+4 b_{2}=0,2 b_{2}+2 c_{2}-d_{2}=0 .
\end{gathered}
$$

Therefore, $a_{1}=b_{1}=0, a_{2}=b_{2}=c_{2}=d_{2}=0$.

From the condition $e_{1} v_{2, k}^{p-3,2}=0$ it follows that $k \leqslant 2$. Since the normal vector $v_{2,2}^{p-3,2}$ cannot be a highest vector, we have that $k=1$. Since

$$
\begin{gathered}
e_{1} v_{2,1}^{p-3,2}=2(p-1) v_{2,0,0,0}+2 v_{0,0,0,0}=0, \\
e_{2} v_{2,1}^{p-3,2}=2 v_{1,0,1,0}-2 v_{0,1,0,0}-2 v_{0,1,0,0}+2 v_{0,1,0,0}=0,
\end{gathered}
$$

we obtain the unique (up to scalar) highest vector $v_{2,1}^{p-3,2}=2 v_{2,0,1,2}-v_{1,1,0,0}-$ $2 v_{0,0,0,1}$.

Since the module $V\left((p-3) \lambda_{1}+2 \lambda_{2}\right)$ has no other highest vectors except $v_{2,1}^{p-3,2}$, the submodule generated by this vector is irreducible. The weight of the highest vector $v_{2,1}^{p-3,2}$ is $(p-3) \lambda_{1}+2 \lambda_{2}-\alpha_{1}-2 \alpha_{2}=(p-3) \lambda_{1}$. Therefore, the maximal submodule of $V\left((p-3) \lambda_{1}+2 \lambda_{2}\right)$ is a module isomorphic to $L\left((p-3) \lambda_{1}\right)$. Analogous calculations show that the vectors

$$
\begin{gathered}
v_{1,1}^{p-2,2}=v_{1,0,1,0}-v_{0,1,0,0}, \\
v_{2,1}^{1, p-2}=2 v_{2,0,1,0}+3 v_{1,1,0,0}-6 v_{0,0,0,1}, \\
v_{1,1}^{1, p-4}=v_{1,0,1,0}+2 v_{0,1,0,0}, \\
v_{p-3, p-3}^{p-2, p-2}=v_{p-3,0, p-3,0}+ \\
\sum_{1 \leqslant j+2 s \leqslant p-2}(-1)^{s}(p-3-j-s) !(p-1) \cdots(p-j-s) v_{p-3-j-2 s, j, p-3-j-s, s}
\end{gathered}
$$


are unique (up to scalar) highest vectors of the modules $V\left((p-2) \lambda_{1}+2 \lambda_{2}\right), V\left(\lambda_{1}+\right.$ $\left.(p-2) \lambda_{2}\right), V\left(\lambda_{1}+(p-4) \lambda_{2}\right), V\left((p-2)\left(\lambda_{1}+\lambda_{2}\right)\right)$ correspondingly.

Therefore, the submodules generated by one of these vectors are irreducible. Their highest weights are respectively $(p-3) \lambda_{1}+2 \lambda_{2}, \lambda_{1}+(p-4) \lambda_{2},(p-4) \lambda_{2}$, $\lambda_{1}+(p-2) \lambda_{2}$. Thus maximal submodules of the following modules $V\left((p-2) \lambda_{1}+2 \lambda_{2}\right)$, $V\left(\lambda_{1}+(p-2) \lambda_{2}\right), V\left(\lambda_{1}+(p-4) \lambda_{2}\right), V\left((p-2)\left(\lambda_{1}+\lambda_{2}\right)\right)$ are the irreducible modules $L\left((p-3) \lambda_{1}+2 \lambda_{2}\right), L\left(\lambda_{1}+(p-4) \lambda_{2}\right), L\left((p-4) \lambda_{2}\right), L\left(\lambda_{1}+(p-2) \lambda_{2}\right)$. The lemma is proved completely.

By analogous methods the following lemma can be proved.

Lemma 3.5. Let $\mathfrak{g}=G_{2}$ and $p>5$. Then

$$
\begin{array}{r}
H^{0}(0)=L(0), H^{0}\left((p-5) \lambda_{1}\right)=L\left((p-5) \lambda_{1}\right), H^{0}\left((p-3) \lambda_{2}\right)= \\
L\left((p-3) \lambda_{2}\right) ; \\
H^{0}\left((p-2) \lambda_{1}+\lambda_{2}\right) / L\left((p-2) \lambda_{1}+\lambda_{2}\right) \cong L\left((p-5) \lambda_{1}+2 \lambda_{2}\right) ; \\
H^{0}\left(3 \lambda_{1}+(p-2) \lambda_{2}\right) / L\left(3 \lambda_{1}+(p-2) \lambda_{2}\right) \cong L\left(4 \lambda_{1}+(p-3) \lambda_{2}\right) ; \\
H^{0}\left((p-5) \lambda_{1}+2 \lambda_{2}\right) / L\left((p-5) \lambda_{1}+2 \lambda_{2}\right) \cong L\left((p-6) \lambda_{1}+2 \lambda_{2}\right) ; \\
H^{0}\left(4 \lambda_{1}+(p-3) \lambda_{2}\right) / L\left(4 \lambda_{1}+(p-3) \lambda_{2}\right) \cong L\left(4 \lambda_{1}+(p-4) \lambda_{2}\right) ; \\
H^{0}\left((p-6) \lambda_{1}+\lambda_{2}\right) / L\left((p-6) \lambda_{1}+\lambda_{2}\right) \cong L\left((p-5) \lambda_{1}\right) ; \\
H^{0}\left(4 \lambda_{1}+(p-4) \lambda_{2}\right) / L\left(4 \lambda_{1}+(p-4) \lambda_{2}\right) \cong L\left((p-3) \lambda_{2}\right) ; \\
H^{0}\left((p-2)\left(\lambda_{1}+\lambda_{2}\right)\right) / L\left((p-2)\left(\lambda_{1}+\lambda_{2}\right)\right) \cong L\left((2 p-6) \lambda_{1}+2 \lambda_{1}\right) .
\end{array}
$$

\section{4. $G_{1}$-cohomology}

Let $S\left(\mathfrak{u}^{*}\right)$ be the symmetric algebra of the Lie algebra $\mathfrak{u}^{*}, w$ an element of Weyl group $W, l(w)$ length of the element $w, \rho$ the half sum of positive roots and $w(\rho)-\rho+p \nu \in X_{1}(T)$. Below we use the following known facts about first cohomology groups of $G_{1}([\mathbf{1 2}]$, proposition 4.9(b) and 4.3) and Andersen-Jantzen general formula $([\mathbf{2}]$, corollary $3.7(\mathrm{a}),(\mathrm{b}))$ :

$$
\begin{gathered}
H^{1}\left(G_{1}, L\left(p \lambda_{i}-\alpha_{i}\right)\right)^{(-1)} \cong H^{0}\left(\lambda_{i}\right), i=1,2, \ldots, n, \\
H^{1}\left(G_{1}, L(\lambda)\right)^{(-1)} \cong\left(H^{0}(\lambda) / L(\lambda)\right)^{G_{1}}, \\
\text { where } \lambda \neq p \lambda_{i}-\alpha_{i}, i=1,2, \ldots, n, \\
H^{i}\left(G_{1}, \mathcal{K}\right)^{(-1)} \cong H^{0}\left(S^{i / 2}\left(\mathfrak{u}^{*}\right)\right) . \\
H^{i}\left(G_{1}, H^{0}\left(\mathcal{K}_{w(\rho)-\rho+p \nu}\right)\right)^{(-1)} \cong H^{0}\left(S^{(i-l(w)) / 2}\left(\mathfrak{u}^{*}\right) \otimes \mathcal{K}_{\nu}\right) .
\end{gathered}
$$


Proposition 4.1. Let $\mathfrak{g}=A_{2}, B_{2}, G_{2}$ and $p>h$. Then $H^{2}\left(G_{1}, H^{0}(\lambda)\right)=0$, except in the following cases

(a) $\mathfrak{g}=A_{2}$

$$
\begin{gathered}
H^{2}\left(G_{1}, H^{0}(0)\right)^{(-1)} \cong \mathfrak{g}^{*} \cong H^{0}\left(\lambda_{1}+\lambda_{2}\right)=L\left(\lambda_{1}+\lambda_{2}\right), \\
H^{2}\left(G_{1}, H^{0}\left((p-3) \lambda_{1}\right)\right)^{(-1)} \cong H^{0}\left(\lambda_{1}\right), \\
H^{2}\left(G_{1}, H^{0}\left((p-3) \lambda_{2}\right)\right)^{(-1)} \cong H^{0}\left(\lambda_{2}\right)
\end{gathered}
$$

(b) $\mathfrak{g}=B_{2}$

$$
\begin{gathered}
H^{2}\left(G_{1}, H^{0}(0)\right)^{(-1)} \cong H^{0}\left(\lambda_{1}\right) \oplus H^{0}\left(2 \lambda_{2}\right), \\
H^{2}\left(G_{1}, H^{0}\left((p-3) \lambda_{1}+2 \lambda_{2}\right)\right)^{(-1)} \cong H^{0}\left(\lambda_{1}\right), \\
H^{2}\left(G_{1}, H^{0}\left(\lambda_{1}+(p-4) \lambda_{2}\right)\right)^{(-1)} \cong H^{0}\left(\lambda_{2}\right)
\end{gathered}
$$

(c) $\mathfrak{g}=G_{2}$

$$
\begin{gathered}
H^{2}\left(G_{1}, H^{0}(0)\right)^{(-1)} \cong H^{0}\left(\lambda_{1}\right) \oplus H^{0}\left(\lambda_{2}\right), \\
H^{2}\left(G_{1}, H^{0}\left((p-5) \lambda_{1}+2 \lambda_{2}\right)\right)^{(-1)} \cong H^{0}\left(\lambda_{1}\right), \\
H^{2}\left(G_{1}, H^{0}\left(4 \lambda_{1}+(p-3) \lambda_{2}\right)\right)^{(-1)} \cong H^{0}\left(\lambda_{2}\right) .
\end{gathered}
$$

Proof. follows from (4) and (5).

For any $\lambda \in X_{1}(T) \backslash\{0\}$ the following exact sequence holds

$$
0 \rightarrow L(\lambda) \rightarrow H^{0}(\lambda) \rightarrow H^{0}(\lambda) / L(\lambda) \rightarrow 0 .
$$

Consider the corresponding long exact sequence of $G_{1}$-cohomology groups

$$
\begin{gathered}
\cdots \rightarrow H^{i}\left(G_{1}, L(\lambda)\right) \rightarrow H^{i}\left(G_{1}, H^{0}(\lambda)\right) \rightarrow H^{i}\left(G_{1}, H^{0}(\lambda) / L(\lambda)\right) \rightarrow \\
H^{i+1}\left(G_{1}, L(\lambda)\right) \rightarrow H^{i+1}\left(G_{1}, H^{0}(\lambda)\right) \rightarrow H^{i+1}\left(G_{1}, H^{0}(\lambda) / L(\lambda)\right) \rightarrow \cdots
\end{gathered}
$$

The triviality of $H^{0}\left(G_{1}, L(\lambda)\right)$ is evident. The module $H^{0}\left(G_{1}, H^{0}(\lambda)\right)$ is an invariant space for $G_{1}$ and a submodule of the $G$-module $H^{0}(\lambda)$. If it is non-zero, it contains the simple socle $L(\lambda)$ of the $G$-module $H^{0}(\lambda)$. Furthermore, $G_{1}$ acts on $L(\lambda)$ in a trivial way if and only if, $\lambda \in p X(T)$. For the restricted weight $\lambda \in X_{1}(T)$ this is possible only in the case $\lambda=0$. Therefore,

$$
H^{0}(\lambda)^{G_{1}}=0
$$

for any $\lambda \in X_{1}(T) \backslash\{0\}$. Then the exact sequence of $G_{1}$-cohomology groups looks like

$$
\begin{array}{r}
0 \rightarrow H^{0}\left(G_{1}, H^{0}(\lambda) / L(\lambda)\right) \rightarrow H^{1}\left(G_{1}, L(\lambda)\right) \rightarrow H^{1}\left(G_{1}, H^{0}(\lambda)\right) \rightarrow \\
H^{1}\left(G_{1}, H^{0}(\lambda) / L(\lambda)\right) \rightarrow H^{2}\left(G_{1}, L(\lambda)\right) \rightarrow H^{2}\left(G_{1}, H^{0}(\lambda)\right) \rightarrow \\
H^{2}\left(G_{1}, H^{0}(\lambda) / L(\lambda)\right) \rightarrow H^{3}\left(G_{1}, L(\lambda)\right) \rightarrow H^{3}\left(G_{1}, H^{0}(\lambda)\right) \rightarrow \cdots
\end{array}
$$


Proposition 4.2. Let $\mathfrak{g}=A_{2}, B_{2}, G_{2}$ and $p>h$. Then $H^{1}\left(G_{1}, L(\lambda)\right)=0$, except in the following cases

(a) $\mathfrak{g}=A_{2}$

$$
\begin{aligned}
& H^{1}\left(G_{1}, L\left((p-2)\left(\lambda_{1}+\lambda_{2}\right)\right)\right)^{(-1)} \cong L(0), \\
& H^{1}\left(G_{1}, L\left((p-2) \lambda_{1}+\lambda_{2}\right)\right)^{(-1)} \cong L\left(\lambda_{1}\right), \\
& H^{1}\left(G_{1}, L\left(\lambda_{1}+(p-2) \lambda_{2}\right)\right)^{(-1)} \cong L\left(\lambda_{2}\right)
\end{aligned}
$$

(b) $\mathfrak{g}=B_{2}$

$$
\begin{gathered}
H^{1}\left(G_{1}, L\left((p-2) \lambda_{1}+2 \lambda_{2}\right)\right)^{(-1)} \cong L\left(\lambda_{1}\right), \\
H^{1}\left(G_{1}, L\left(\lambda_{1}+(p-2) \lambda_{2}\right)\right)^{(-1)} \cong L\left(\lambda_{2}\right) ;
\end{gathered}
$$

(c) $\mathfrak{g}=G_{2}$

$$
\begin{gathered}
H^{1}\left(G_{1}, L\left((p-2) \lambda_{1}+\lambda_{2}\right)\right)^{(-1)} \cong L\left(\lambda_{1}\right), \\
H^{1}\left(G_{1}, L\left(3 \lambda_{1}+(p-2) \lambda_{2}\right)\right)^{(-1)} \cong L\left(\lambda_{2}\right) .
\end{gathered}
$$

Proof. As we mentioned above, the first ordinary cohomology groups and the corresponding cohomology groups for $G_{1}$ coincide. Statement (a) was proved in ([5],(3.6), p.112) and ([12], 6.10, p.314).

We now prove (b) and (c). We will use (2) and (3). Let us consider the induced modules $H^{0}(\lambda)$ corresponding to the weights from the list of corollary 3.2.

By lemmas 3.4 and 3.5 the factor-modules $H^{0}(\lambda) / L(\lambda)$ for the Lie algebras $\mathfrak{g}=$ $B_{2}, G_{2}$ are simple and the highest weight of $H^{0}(\lambda) / L(\lambda)$ is not an element of $p X(T)$, therefore $\left(H^{0}(\lambda) / L(\lambda)\right)^{G_{1}}=0$ for peculiar modules. So, nontrivial first cohomology groups can appear only for modules of the form $L\left(p \lambda_{i}-\alpha_{i}\right)$ and they are given by (2).

Let $\mathfrak{g}=B_{2}$. We have $p \lambda_{1}-\alpha_{1}=p \lambda_{1}-2 \lambda_{1}+\lambda_{2}=(p-2) \lambda_{1}+\lambda_{2}, p \lambda_{2}-\alpha_{2}=$ $p \lambda_{2}+\lambda_{1}-2 \lambda_{2}=\lambda_{1}+(p-2) \lambda_{2}$. So, according to (2) we obtain (b).

If $\mathfrak{g}=G_{2}$, then $p \lambda_{1}-\alpha_{1}=p \lambda_{1}-2 \lambda_{1}+\lambda_{2}=(p-2) \lambda_{1}+\lambda_{2}, p \lambda_{2}-\alpha_{2}=$ $p \lambda_{2}+3 \lambda_{1}-2 \lambda_{2}=3 \lambda_{1}+(p-2) \lambda_{2}$. So, by (2) we obtain (c).

Proposition 4.3. Let $\mathfrak{g}=A_{2}, B_{2}, G_{2}$ and $p>h$. Then $H^{2}\left(G_{1}, L(\lambda)\right)=0$, except in the following cases

(a) $\mathfrak{g}=A_{2}$

$$
\begin{gathered}
H^{2}\left(G_{1}, L(0)\right)^{(-1)} \cong \mathfrak{g}^{*} \cong H^{0}\left(\lambda_{1}+\lambda_{2}\right)=L\left(\lambda_{1}+\lambda_{2}\right), \\
H^{2}\left(G_{1}, L\left((p-3) \lambda_{1}\right)\right)^{(-1)} \cong L\left(\lambda_{1}\right), \\
H^{2}\left(G_{1}, L\left((p-3) \lambda_{2}\right)\right)^{(-1)} \cong L\left(\lambda_{2}\right) ;
\end{gathered}
$$

(b) $\mathfrak{g}=B_{2}$

$$
\begin{gathered}
H^{2}\left(G_{1}, L(0)\right)^{(-1)} \cong L\left(\lambda_{1}\right) \oplus L\left(2 \lambda_{2}\right), \\
H^{2}\left(G_{1}, L\left((p-3) \lambda_{1}+2 \lambda_{2}\right)\right)^{(-1)} \cong L\left(\lambda_{1}\right), \\
H^{2}\left(G_{1}, L\left(\lambda_{1}+(p-4) \lambda_{2}\right)\right)^{(-1)} \cong L\left(\lambda_{2}\right)
\end{gathered}
$$




$$
H^{2}\left(G_{1}, L\left((p-2)\left(\lambda_{1}+\lambda_{2}\right)\right)^{(-1)} \cong L\left(\lambda_{2}\right)\right.
$$

(c) $\mathfrak{g}=G_{2}$

$$
\begin{gathered}
H^{2}\left(G_{1}, L(0)\right)^{(-1)} \cong L\left(\lambda_{1}\right) \oplus L\left(\lambda_{2}\right), \\
H^{2}\left(G_{1}, L\left((p-5) \lambda_{1}+2 \lambda_{2}\right)\right)^{(-1)} \cong L\left(\lambda_{1}\right), \\
H^{2}\left(G_{1}, L\left(4 \lambda_{1}+(p-3) \lambda_{2}\right)\right)^{(-1)} \cong L\left(\lambda_{2}\right) .
\end{gathered}
$$

Proof. (a) follows from the exact sequence (8), lemma 3.3 and propositions 4.1, 4.2, part (a).

(b) follows from the exact sequence (8), lemma 3.4 and propositions 4.1 and 4.2 , part (b).

(c) follows from the exact sequence (8), lemma 3.5 and propositions 4.1 and 4.2 , part (c).

\section{5. $\mathfrak{g}$-cohomology}

To prove theorem 1.1 we need some lemmas.

Lemma 5.1. Let $\mathfrak{g}$ be a Lie algebra, $V$ be a restricted $\mathfrak{g}$-module. For an associative 2-cocycle $\psi$, let $\psi^{\prime}$ be the function defined by $\psi_{x}^{\prime}(y)=\psi\left(x^{p}-x^{[p]}, y\right)-\psi\left(y, x^{p}-x^{[p]}\right)$. Then the map $\psi \rightarrow \psi^{\prime}$ induces a $\mathcal{K}$-linear map of $H^{2}(\mathfrak{g}, V)$ into $S\left(\mathfrak{g}, H^{1}(\mathfrak{g}, V)\right)$.

Proof. [9], Theorem 3.1.

Lemma 5.2. Let $\mathfrak{g}=A_{2}, B_{2}, G_{2}$. Suppose that $V$ is a restricted irreducible $\mathfrak{g}$-module and $H^{1}(\mathfrak{g}, V) \neq 0$. Then the lists of possible weights of the $G$-module $H^{2}(\mathfrak{g}, V)$ and the lists of possible dominant weights are the following

$$
\begin{array}{llll}
\mathfrak{g} & V & \text { weights of } H^{2}(\mathfrak{g}, V) & \text { dominants } \\
& & & \\
A_{2} & L\left((p-2) \lambda_{1}+\lambda_{2}\right) & p \lambda_{1}, p\left(-\lambda_{1}+\lambda_{2}\right), p\left(-\lambda_{2}\right) & p \lambda_{1} \\
A_{2} & L\left(\lambda_{1}+(p-2) \lambda_{2}\right) & p \lambda_{2}, p\left(\lambda_{1}-\lambda_{2}\right),-p \lambda_{1} & p \lambda_{2} \\
A_{2} & L\left((p-2)\left(\lambda_{1}+\lambda_{2}\right)\right) & 0, p\left(2 \lambda_{1}-\lambda_{2}\right), p\left(-\lambda_{1}+2 \lambda_{2}\right), & p\left(\lambda_{1}+\lambda_{2}\right) \\
& & p\left(\lambda_{1}+\lambda_{2}\right), p\left(-2 \lambda_{1}+\lambda_{2}\right), & \\
& & p\left(\lambda_{1}-2 \lambda_{2}\right), p\left(-\lambda_{1}-\lambda_{2}\right) & \\
B_{2} & L\left((p-2) \lambda_{1}+2 \lambda_{2}\right) & 0, p \lambda_{1}-p \lambda_{1}, p\left(-\lambda_{1}+2 \lambda_{2}\right), & \\
& & p\left(\lambda_{1}-2 \lambda_{2}\right) & \\
B_{2} & L\left(\lambda_{1}+(p-2) \lambda_{2}\right) & p \lambda_{2}-p \lambda_{2}, p\left(\lambda_{1}-\lambda_{2}\right) & \\
& & p\left(-\lambda_{1}+\lambda_{2}\right) & \\
G_{2} & L\left((p-2) \lambda_{1}+\lambda_{2}\right) & 0, p \lambda_{1},-p \lambda_{1}, p\left(-\lambda_{1}+\lambda_{2}\right), & \\
& & p\left(2 \lambda_{1}-\lambda_{2}\right), p\left(\lambda_{1}-\lambda_{2}\right), & \\
& & p\left(-2 \lambda_{1}+\lambda_{2}\right) & \\
G_{2} & L\left(3 \lambda_{1}+(p-2) \lambda_{2}\right) & 0, p \lambda_{2},-p \lambda_{2}, p\left(2 \lambda_{1}-\lambda_{2}\right), & \\
& & p\left(-3 \lambda_{1}+2 \lambda_{2}\right), p\left(-\lambda_{1}+\lambda_{2}\right), & \\
& & p\left(3 \lambda_{1}-\lambda_{2}\right), p \lambda_{1}, p\left(-2 \lambda_{1}+\lambda_{2}\right), & \\
& & p\left(3 \lambda_{1}-2 \lambda_{2}\right), p\left(\lambda_{1}-\lambda_{2}\right), & \\
& p\left(-3 \lambda_{1}+\lambda_{2}\right),-p \lambda_{1} &
\end{array}
$$


Proof. It follows from lemma 5.1 and proposition 4.2.

Lemma 5.3. Let $\mathfrak{g}=B_{2}$ and $p \geqslant 5$. Then

$$
H^{3}\left(G_{1}, L\left((p-3) \lambda_{1}+2 \lambda_{2}\right)\right)=H^{3}\left(G_{1}, L\left(\lambda_{1}+(p-4) \lambda_{2}\right)\right)=0 .
$$

Proof. By lemma 3.4 the following sequence is exact

$$
0 \rightarrow L\left((p-3) \lambda_{1}+2 \lambda_{2}\right) \rightarrow H^{0}\left((p-3) \lambda_{1}+2 \lambda_{2}\right) \rightarrow L\left((p-3) \lambda_{1}\right) \rightarrow 0 .
$$

The corresponding exact sequence of $G_{1}$-cohomology groups gives us that the following sequence is exact

$$
\begin{array}{r}
H^{2}\left(G_{1}, L\left((p-3) \lambda_{1}\right)\right) \rightarrow H^{3}\left(G_{1}, L\left((p-3) \lambda_{1}+2 \lambda_{2}\right)\right) \rightarrow \\
H^{3}\left(G_{1}, H^{0}\left((p-3) \lambda_{1}+2 \lambda_{2}\right)\right)
\end{array}
$$

By (5) $H^{3}\left(G_{1}, H^{0}\left((p-3) \lambda_{1}+2 \lambda_{2}\right)\right)=0$, since $i=3, l(w)=2$. By proposition 4.3 we have $H^{2}\left(G_{1}, L\left((p-3) \lambda_{1}\right)\right)=0$. Therefore, from the exact sequence (9) we obtain $H^{3}\left(G_{1}, L\left((p-3) \lambda_{1}+2 \lambda_{2}\right)\right)=0$.

The second statement $H^{3}\left(G_{1}, L\left(\lambda_{1}+(p-4) \lambda_{2}\right)\right)=0$ can be proved in an analogous way.

Lemma 5.4. Let $\mathfrak{g}=G_{2}$ and $p>5$. Then

$$
H^{3}\left(G_{1}, L\left((p-5) \lambda_{1}+2 \lambda_{2}\right)\right)=0 .
$$

Proof. By lemma 3.5 the following sequence is exact

$$
0 \rightarrow L\left((p-5) \lambda_{1}+2 \lambda_{2}\right) \rightarrow H^{0}\left((p-5) \lambda_{1}+2 \lambda_{2}\right) \rightarrow L\left((2 p-6) \lambda_{1}+2 \lambda_{2}\right) \rightarrow 0 .
$$

Therefore, the following sequence of $G_{1}$-cohomology groups is exact

$$
\begin{gathered}
H^{2}\left(G_{1}, L\left((2 p-6) \lambda_{1}+2 \lambda_{2}\right)\right) \rightarrow H^{3}\left(G_{1}, L\left((p-5) \lambda_{1}+2 \lambda_{2}\right)\right) \rightarrow \\
H^{3}\left(G_{1}, H^{0}\left((p-5) \lambda_{1}+2 \lambda_{2}\right)\right)
\end{gathered}
$$

By $(5) H^{3}\left(G_{1}, H^{0}\left((p-5) \lambda_{1}+2 \lambda_{2}\right)\right)=0$, since $i=3, l(w)=2$. By proposition 4.3 $H^{2}\left(G_{1}, L\left((2 p-6) \lambda_{1}+2 \lambda_{2}\right)\right)=0$. Then from the exact sequence (10) it follows that $H^{3}\left(G_{1}, L\left((p-5) \lambda_{1}+2 \lambda_{2}\right)\right)=0$.

Proof of theorem 1.1. The proof is divided into two parts. In the first part we prove all isomorphisms mentioned in theorem 1.1. In the second part we establish that for all other weights given in corollary 3.2 the second cohomology groups are trivial.

Part 1. By lemma 2.1 all isomorphisms, except the case $\mathfrak{g}=G_{2}$ and $V=$ $L\left(3 \lambda_{1}+(p-2) \lambda_{2}\right)$, follow from proposition 4.3.

Let us prove the last isomorphism of (c). If $H^{2}\left(G_{1}, L(\lambda)\right)=0$, then from the exact sequence (1) it follows that $H^{2}(\mathfrak{g}, L(\lambda))$ is isomorphic to the kernel of the map

$$
f: H^{1}(\mathfrak{g}, L(\lambda)) \otimes \mathfrak{g}^{*} \rightarrow H^{3}\left(G_{1}, L(\lambda)\right) .
$$

By proposition $4.3 H^{2}\left(G_{1}, L\left(3 \lambda_{1}+(p-2) \lambda_{2}\right)\right)=0$. Hence $H^{2}\left(\mathfrak{g}, L\left(3 \lambda_{1}+(p-2) \lambda_{2}\right)\right)$ is isomorphic to ker $f$. By (5)

$$
H^{3}\left(G_{1}, H^{0}\left(3 \lambda_{1}+(p-2) \lambda_{2}\right)\right)^{(-1)} \cong
$$




$$
H^{0}\left(2 \lambda_{1}\right) \oplus H^{0}\left(2 \lambda_{2}\right) \oplus H^{0}\left(3 \lambda_{1}\right) \oplus H^{0}\left(\lambda_{1}+\lambda_{2}\right) .
$$

By lemma $3.5 H^{0}\left(3 \lambda_{1}+(p-2) \lambda_{2}\right) / L\left(3 \lambda_{1}+(p-2) \lambda_{2}\right) \cong L\left(4 \lambda_{1}+(p-3) \lambda_{2}\right)$ and by proposition $4.3 H^{2}\left(G_{1}, L\left(4 \lambda_{1}+(p-3) \lambda_{2}\right)\right)^{(-1)} \cong L\left(\lambda_{2}\right)$. Therefore, by the exact sequence (8), $H^{3}\left(G_{1}, L\left(3 \lambda_{1}+(p-2) \lambda_{2}\right)\right)$ as a $G$-module has (possible) composition factors $H^{0}\left(2 \lambda_{1}\right), H^{0}\left(2 \lambda_{2}\right), H^{0}\left(3 \lambda_{2}\right), H^{0}\left(\lambda_{1}+\lambda_{2}\right)$ and $H^{0}\left(\lambda_{2}\right)$.

By proposition $4.2 H^{1}\left(\mathfrak{g}, L\left(3 \lambda_{1}+(p-2) \lambda_{2}\right)\right) \cong L\left(\lambda_{2}\right)$. Therefore,

$$
\begin{gathered}
H^{1}\left(\mathfrak{g}, L\left(3 \lambda_{1}+(p-2) \lambda_{2}\right)\right) \otimes \mathfrak{g}^{*} \cong L\left(\lambda_{2}\right) \otimes \mathfrak{g} \cong \\
H^{0}\left(2 \lambda_{1}\right) \oplus H^{0}\left(2 \lambda_{2}\right) \oplus H^{0}\left(3 \lambda_{1}\right) \oplus H^{0}\left(\lambda_{2}\right) \oplus H^{0}(0) .
\end{gathered}
$$

From the decompositions of $H^{1}\left(\mathfrak{g}, L\left(3 \lambda_{1}+(p-2) \lambda_{2}\right)\right) \otimes \mathfrak{g}^{*}$ and $H^{3}\left(G_{1}, L\left(3 \lambda_{1}+\right.\right.$ $\left.\left.(p-2) \lambda_{2}\right)\right)$ we obtain that $H^{0}(0)=L(0) \subseteq \operatorname{ker} f$.

If ker $f$ contains some of the $G$-modules $H^{0}\left(2 \lambda_{1}\right), H^{0}\left(2 \lambda_{2}\right)$, $H^{0}\left(3 \lambda_{1}\right)$ and $H^{0}\left(\lambda_{2}\right)$, then the $G$-module $H^{2}\left(\mathfrak{g}, L\left(3 \lambda_{1}+(p-2) \lambda_{2}\right)\right)$ has nontrivial elements with weights $2 p \lambda_{1}, 2 p \lambda_{2}, 3 p \lambda_{1}$, or $p \lambda_{2}$. We will prove that this is impossible.

For $\mathfrak{g}=G_{2}$ the list of dominant weights of adjoint $G$-module is $\left\{0, p \lambda_{1}, p \lambda_{2}\right\}$ (see lemma 5.2). Therefore, the only non-zero dominant weights of the $G$-module $H^{2}\left(\mathfrak{g}, L\left(3 \lambda_{1}+(p-2) \lambda_{2}\right)\right)$ are $p \lambda_{1}$ or $p \lambda_{2}$. Thus cocycles in $Z^{2}\left(\mathfrak{g}, L\left(3 \lambda_{1}+(p-2) \lambda_{2}\right)\right)$ with weights $2 p \lambda_{1}, 2 p \lambda_{2}$,

$3 p \lambda_{1}$ are coboundaries.

Now we prove that the classes of cocycles with weights $p \lambda_{2}$ are also trivial. To do it we use the realization of the $\mathfrak{g}$-module $L\left(3 \lambda_{1}+(p-2) \lambda_{2}\right)$ as a factor-module of the Weyl module.

Let $f_{i}, h_{j}, e_{i}: i=1, \ldots, 6, j=1,2$ be a Chevalley basis of $\mathfrak{g}$, where $f_{i}=$ $e_{-\alpha_{i}}, e_{i}=e_{\alpha_{i}}$ for $i=1,2$ i $f_{3}=e_{-\alpha_{1}-\alpha_{2}}, f_{4}=e_{-2 \alpha_{1}-\alpha_{2}}, f_{5}=e_{-3 \alpha_{1}-\alpha_{2}}, f_{6}=$ $e_{-3 \alpha_{1}-2 \alpha_{2}}, e_{3}=e_{\alpha_{1}+\alpha_{2}}, e_{4}=e_{2 \alpha_{1}+\alpha_{2}}, e_{5}=e_{3 \alpha_{1}+\alpha_{2}}, e_{6}=e_{3 \alpha_{1}+2 \alpha_{2}}$. The Weyl module $V\left(m_{1} \lambda_{1}+m_{2} \lambda_{2}\right)$ can be defined on the vector space

$$
v_{i, j, k, l, s, t}:=\frac{f_{6}^{t} f_{5}^{s} f_{4}^{l} f_{3}^{k} f_{2}^{j} f_{1}^{i}}{t ! s ! l ! k ! j ! i !} \otimes v_{m_{1} \lambda_{1}+m_{2} \lambda_{2}},
$$

where $v_{m_{1} \lambda_{1}+m_{2} \lambda_{2}}$ is the highest weight, by

$$
\begin{gathered}
e_{1} v_{i, j, k, l, s, t}=(l+1) v_{i, j, k+1, l, s-1, t}-3(t+1) v_{i, j, k, l-2, s, t+1}- \\
2(k+1) v_{i, j, k+1, l-1, s, t}-3(j+1) v_{i, j+1, k-1, l, s, t}+\left(m_{1}+1-i\right) v_{i-1, j, k, l, s, t}, \\
e_{2} v_{i, j, k, l, s, t}=(s+1) v_{i, j, k, l, s+1, t-1}-(l+1) v_{i, j, k-2, l+1, s, t}+ \\
(i+1) v_{i+1, j, k-1, l, s, t}-2(t+1) v_{i, j, k-3, l, s, t+1}+ \\
\left(m_{2}+1+i-j-k\right) v_{i, j-1, k, l, s, t}, \\
f_{1} v_{i, j, k, l, s, t}=-3(s+1) v_{i, j, k, l-1, s+1, t}-3(t+1) v_{i, j, k-2, l, s, t+1} \\
-2(l+1) v_{i, j, k-1, l+1, s, t}-(k+1) v_{i, j-1, k+1, l, s, t}+(i+1) v_{i+1, j, k, l, s, t},
\end{gathered}
$$




$$
f_{2} v_{i, j, k, l, s, t}=-(t+1) v_{i, j, k, l, s-1, t+1}+(j+1) v_{i, j+1, k, l, s, t} .
$$

If some cocycle $\psi$ of the module $L\left(3 \lambda_{1}+(p-2) \lambda_{2}\right)$ has weight $p \lambda_{2}$, then

$$
\begin{aligned}
& \psi\left(e_{1}, f_{3}\right)=x_{1} v_{0,0,0,0,0,0}, \quad \psi\left(e_{5}, f_{6}\right)=x_{2} v_{0,0,0,0,0,0}, \\
& \psi\left(f_{1}, f_{2}\right)=x_{3} v_{1,0,0,0,0,0}, \quad \psi\left(e_{1}, f_{4}\right)=x_{4} v_{1,0,0,0,0,0}, \\
& \psi\left(e_{4}, f_{6}\right)=x_{5} v_{1,0,0,0,0,0}, \quad \psi\left(f_{1}, f_{3}\right)=x_{6} v_{2,0,0,0,0,0}, \\
& \psi\left(e_{1}, f_{5}\right)=x_{7} v_{2,0,0,0,0,0}, \quad \psi\left(e_{3}, f_{6}\right)=x_{8} v_{2,0,0,0,0,0}, \\
& \psi\left(f_{1}, f_{4}\right)=x_{9} v_{3,0,0,0,0,0}, \quad \psi\left(e_{2}, f_{6}\right)=x_{10} v_{3,0,0,0,0,0}, \\
& \psi\left(f_{2}, f_{3}\right)=x_{11} v_{1,1,0,0,0,0}+x_{12} v_{0,0,1,0,0,0}, \\
& \psi\left(f_{1}, f_{6}\right)=x_{13} v_{3,0,1,0,0,0}+x_{14} v_{2,0,0,1,0,0}+x_{15} v_{1,0,0,0,1,0}, \\
& \psi\left(e_{1}, f_{6}\right)=x_{16} v_{2,1,0,0,0,0}+x_{17} v_{1,0,1,0,0,0}+x_{18} v_{0,0,0,1,0,0}, \\
& \psi\left(f_{2}, f_{4}\right)=x_{19} v_{2,1,0,0,0,0}+x_{20} v_{1,0,1,0,0,0}+x_{21} v_{0,0,0,1,0,0}, \\
& \psi\left(f_{2}, f_{5}\right)=x_{22} v_{3,1,0,0,0,0}+x_{23} v_{2,0,1,0,0,0}+x_{24} v_{1,0,0,1,0,0}+x_{25} v_{0,0,0,0,1,0}, \\
& \psi\left(f_{3}, f_{4}\right)=x_{26} v_{3,1,0,0,0,0}+x_{27} v_{2,0,1,0,0,0}+x_{28} v_{1,0,0,1,0,0}+x_{29} v_{0,0,0,0,1,0}, \\
& \psi\left(f_{2}, f_{6}\right)=x_{30} v_{3,2,0,0,0,0}+x_{31} v_{2,1,1,0,0,0}+x_{32} v_{1,0,2,0,0,0}+x_{33} v_{1,1,0,1,0,0}+ \\
& x_{34} v_{0,0,1,1,0,0}+x_{35} v_{0,1,0,0,1,0}+x_{36} v_{0,0,0,0,0,1}, \\
& \psi\left(f_{3}, f_{5}\right)=x_{37} v_{3,0,1,0,0,0}+x_{38} v_{2,0,0,1,0,0}+x_{39} v_{1,0,0,0,1,0}, \\
& \psi\left(f_{3}, f_{6}\right)=x_{40} v_{3,1,1,0,0,0}+x_{41} v_{2,0,2,0,0,0}+x_{42} v_{2,1,0,1,0,0}+x_{43} v_{0,0,0,2,0,0}+ \\
& x_{44} v_{1,0,1,1,0,0}+x_{45} v_{1,1,0,0,1,0}+x_{46} v_{0,0,1,0,1,0}+x_{47} v_{1,0,0,0,0,1}, \\
& \psi\left(f_{4}, f_{5}\right)=x_{48} v_{3,0,0,1,0,0}+x_{49} v_{2,0,0,0,1,0}, \\
& \psi\left(f_{4}, f_{6}\right)=x_{50} v_{3,0,2,0,0,0}+x_{51} v_{3,1,0,1,0,0}+x_{52} v_{1,0,0,2,0,0}+x_{53} v_{2,0,1,1,0,0}+ \\
& x_{54} v_{2,1,0,0,1,0}+x_{55} v_{1,0,1,0,1,0}+x_{56} v_{0,0,0,1,1,0}+x_{57} v_{2,0,0,0,0,1}, \\
& \psi\left(f_{5}, f_{6}\right)=x_{58} v_{3,0,1,1,0,0}+x_{59} v_{2,0,0,2,0,0}+x_{60} v_{3,1,0,0,1,0}+x_{61} v_{0,0,0,0,2,0}+ \\
& x_{62} v_{2,0,1,0,1,0}+x_{63} v_{1,0,0,1,1,0}+x_{64} v_{3,0,0,0,0,1},
\end{aligned}
$$

for some $x_{i} \in \mathcal{K} \quad(i=1, \ldots, 64)$. Non-written components of $\psi(X, Y)$ are equal to 0 (sums of weights of $X$ and $Y$ can not be a weight of the module).

The conditions

$$
d \psi\left(h_{i}, f_{2}, f_{6}\right)=0, d \psi\left(h_{i}, f_{2}, f_{5}\right)=0, d \psi\left(h_{i}, e_{1}, f_{3}\right)=0, i=1,2,
$$


gives us a system of linear equations. Solving this system (we omit the standard but long calculations) gives us that

$$
\psi\left(h_{i}, f_{2}\right)=\psi\left(h_{i}, f_{3}\right)=\psi\left(h_{i}, f_{4}\right)=\psi\left(h_{i}, f_{5}\right)=\psi\left(h_{i}, f_{6}\right)=0, i=1,2 .
$$

Furthermore, from the following system of linear equations with 16 conditions

$$
\begin{array}{r}
d \psi\left(f_{4}, f_{5}, f_{6}\right)=0, d \psi\left(f_{3}, f_{5}, f_{6}\right)=0, d \psi\left(f_{3}, f_{4}, f_{5}\right)=0, \\
d \psi\left(f_{2}, f_{4}, f_{5}\right)=0, d \psi\left(f_{2}, f_{5}, f_{6}\right)=0, d \psi\left(f_{1}, f_{4}, f_{6}\right)=0, \\
d \psi\left(e_{1}, f_{5}, f_{6}\right)=0, d \psi\left(e_{2}, f_{5}, f_{6}\right)=0, d \psi\left(e_{3}, f_{5}, f_{6}\right)=0, \\
d \psi\left(e_{4}, f_{5}, f_{6}\right)=0, d \psi\left(e_{5}, f_{4}, f_{5}\right)=0, d \psi\left(e_{6}, f_{5}, f_{6}\right)=0, \\
d \psi\left(e_{2}, f_{4}, f_{6}\right)=0, d \psi\left(e_{3}, f_{3}, f_{4}\right)=0, d \psi\left(e_{1}, f_{3}, f_{4}\right)=0, \\
d \psi\left(e_{1}, f_{4}, f_{6}\right)=0
\end{array}
$$

we obtain that $\psi$ should have the following form

$$
\begin{gathered}
\psi\left(f_{1}, f_{2}\right)=x_{3} v_{1,0,0,0,0,0}, \psi\left(f_{1}, f_{3}\right)=x_{6} v_{2,0,0,0,0,0}, \\
\psi\left(f_{2}, f_{3}\right)=x_{11} v_{1,1,0,0,0,0}+x_{12} v_{0,0,1,0,0,0} .
\end{gathered}
$$

Then from the equation $d \psi\left(e_{1}, f_{1}, f_{2}\right)=0$ we obtain that $x_{3}=0$. Since $\psi\left(f_{1}, f_{2}\right)=$ 0 , then the condition $d \psi\left(e_{1}, f_{1}, f_{3}\right)=0$ gives us that $x_{6}=0$. Finally, from the condition $d \psi\left(f_{2}, f_{3}, f_{6}\right)=0$, we have that $\psi\left(f_{2}, f_{3}\right)=0$. Therefore, any cocycle with weight $p \lambda_{1}$ is trivial.

So, in the case $L(\lambda)=L\left(3 \lambda_{1}+(p-2) \lambda_{2}\right)$, the kernel of $f$ is isomorphic to the $G$-module $H^{0}(0)=L(0)$. Thus,

$$
H^{2}\left(G_{2}, L\left(3 \lambda_{1}+(p-2) \lambda_{2}\right)\right) \cong L(0)^{(1)} .
$$

Part 2. We now prove that the second cohomology groups of irreducible peculiar modules which are not mentioned in the formulation of theorem 1.1 are trivial.

The lists of highest weights of peculiar modules are given in corollary 3.2. By proposition 4.3 the second cohomology groups of $G_{1}$ for these modules are trivial. Therefore, it is enough to show that ker $f=0$ for any of these modules. We will do it by considering each case of algebras separately.

The case (a). By proposition 4.2 only the following 3 modules have nontrivial first cohomology groups $L\left((p-2)\left(\lambda_{1}+\lambda_{2}\right)\right), L\left((p-2) \lambda_{1}+\lambda_{2}\right), L\left(\lambda_{1}+(p-2) \lambda_{2}\right)$. By (4) and (5) we have

$$
\begin{gathered}
H^{3}\left(G_{1}, H^{0}\left((p-2)\left(\lambda_{1}+\lambda_{2}\right)\right)\right)^{(-1)} \cong H^{0}\left(\lambda_{1}+\lambda_{2}\right), \\
H^{3}\left(G_{1}, H^{0}\left((p-2) \lambda_{1}+\lambda_{2}\right)\right)^{(-1)} \cong H^{0}\left(2 \lambda_{2}\right) \oplus H^{0}\left(2 \lambda_{1}+\lambda_{2}\right), \\
H^{3}\left(G_{1}, H^{0}\left(\lambda_{1}+(p-2) \lambda_{2}\right)\right)^{(-1)} \cong H^{0}\left(2 \lambda_{1}\right) \oplus H^{0}\left(\lambda_{1}+2 \lambda_{2}\right) . \\
H^{3}\left(G_{1}, H^{0}(\lambda)\right)^{(-1)}=0 \text { if } \lambda=0,(p-3) \lambda_{1},(p-3) \lambda_{2} .
\end{gathered}
$$


According to (14)-(17), lemma 3.3 and proposition 4.3 the exact sequence (8) gives us the following exact sequences

$$
\begin{gathered}
0 \rightarrow H^{0}\left(\lambda_{1}+\lambda_{2}\right) \rightarrow H^{3}\left(G_{1}, L\left((p-2)\left(\lambda_{1}+\lambda_{2}\right)\right)\right) \rightarrow H^{0}\left(\lambda_{1}+\lambda_{2}\right) \rightarrow 0, \\
0 \rightarrow L\left(\lambda_{1}\right) \rightarrow H^{3}\left(G_{1}, L\left((p-2) \lambda_{1}+\lambda_{2}\right)\right) \rightarrow \\
H^{0}\left(2 \lambda_{2}\right) \oplus H^{0}\left(2 \lambda_{1}+\lambda_{2}\right) \rightarrow 0, \\
0 \rightarrow L\left(\lambda_{2}\right) \rightarrow H^{3}\left(G_{1}, L\left(\lambda_{1}+(p-2) \lambda_{2}\right)\right) \rightarrow \\
H^{0}\left(2 \lambda_{1}\right) \oplus H^{0}\left(\lambda_{1}+2 \lambda_{2}\right) \rightarrow 0 .
\end{gathered}
$$

On the other hand, according to proposition 4.2 , we have

$$
\begin{gathered}
H^{1}\left(\mathfrak{g}, L\left((p-2)\left(\lambda_{1}+\lambda_{2}\right)\right) \otimes \mathfrak{g}^{*} \cong L(0) \otimes \mathfrak{g} \cong \mathfrak{g} \cong H^{0}\left(\lambda_{1}+\lambda_{2}\right),\right. \\
H^{1}\left(\mathfrak{g}, L\left((p-2) \lambda_{1}+\lambda_{2}\right)\right) \otimes \mathfrak{g}^{*} \cong L\left(\lambda_{1}\right) \otimes \mathfrak{g} \cong \\
H^{0}\left(2 \lambda_{2}\right) \oplus H^{0}\left(2 \lambda_{1}+\lambda_{2}\right) \oplus H^{0}\left(\lambda_{1}\right), \\
H^{1}\left(g, L\left(\lambda_{1}+(p-2) \lambda_{2}\right)\right) \otimes \mathfrak{g}^{*} \cong \\
L\left(\lambda_{2}\right) \otimes \mathfrak{g} \cong H^{0}\left(2 \lambda_{1}\right) \oplus H^{0}\left(\lambda_{1}+2 \lambda_{2}\right) \oplus H^{0}\left(\lambda_{2}\right) .
\end{gathered}
$$

Consider each case of these modules.

Let $V=L\left((p-2)\left(\lambda_{1}+\lambda_{2}\right)\right)$. From (14) and (21) we see that $H^{3}\left(G_{1}, L((p-\right.$ $\left.2)\left(\lambda_{1}+\lambda_{2}\right)\right)$ contains a composition factor $H^{0}\left(\lambda_{1}+\lambda_{2}\right)$ isomorphic to $H^{1}(\mathfrak{g}, L((p-$ 2) $\left.\left.\left(\lambda_{1}+\lambda_{2}\right)\right)\right) \otimes \mathfrak{g}^{*}$.

If ker $f$ contains $H^{0}\left(\lambda_{1}+\lambda_{2}\right)$, then the second cohomology group $H^{2}(\mathfrak{g}, L((p-$ $\left.\left.2)\left(\lambda_{1}+\lambda_{2}\right)\right)\right)$ as a $G$-module contains classes of cocycles of weights $p\left(\lambda_{1}+\lambda_{2}\right)$. The highest weight $p\left(\lambda_{1}+\lambda_{2}\right)-2 \alpha_{1}-2 \alpha_{2}$ can not be presented as a sum of $p\left(\lambda_{1}+\lambda_{2}\right)$ and two roots. Therefore, such a case is impossible. Therefore, $H^{0}\left(\lambda_{1}+\lambda_{2}\right)$ can not be in ker $f$. Then the exact sequence (1) for $L\left((p-2)\left(\lambda_{1}+\lambda_{2}\right)\right)$ gives us that ker $f=0$.

Let $f_{i}, h_{j}, e_{i}: i=1,2,3, j=1,2$ be the Chevalley basis of $\mathfrak{g}$, where $f_{i}=$ $e_{-\alpha_{i}}, e_{i}=e_{\alpha_{i}}$ for $i=1,2$ and $f_{3}=e_{-\alpha_{1}-\alpha_{2}}, e_{3}=e_{\alpha_{1}+\alpha_{2}}$. The Weyl module $V\left(m_{1} \lambda_{1}+m_{2} \lambda_{2}\right)$ can be defined on the vector space with basis

$$
v_{i, j, k}:=\frac{f_{3}^{k} f_{2}^{j} f_{1}^{i}}{k ! j ! i !} \otimes v_{m_{1} \lambda_{1}+m_{2} \lambda_{2}}
$$

where $v_{m_{1} \lambda_{1}+m_{2} \lambda_{2}}$ is the highest weight.

The action of $\mathfrak{g}$ is given by

$$
e_{1} v_{i, j, k}=-(j+1) v_{i, j+1, k-1}+\left(m_{1}+1-i\right) v_{i-1, j, k},
$$




$$
\begin{gathered}
e_{2} v_{i, j, k}=(i+1) v_{i+1, j, k-1}+\left(m_{2}+1+i-j-k\right) v_{i, j-1, k}, \\
f_{1} v_{i, j, k}=-(k+1) v_{i, j-1, k+1}, \\
f_{2} v_{i, j, k}=(j+1) v_{i, j+1, k} .
\end{gathered}
$$

Let now $V=L\left((p-2) \lambda_{1}+\lambda_{2}\right)$. From (19) and (22) we see that $H^{1}(\mathfrak{g}, L((p-$ 2) $\left.\left.\lambda_{1}+\lambda_{2}\right)\right) \otimes \mathfrak{g}^{*}$ and $H^{3}\left(G_{1}, L\left((p-2) \lambda_{1}+\lambda_{2}\right)\right)$ have isomorphic composition factors $H^{0}\left(2 \lambda_{1}+\lambda_{2}\right), H^{0}\left(2 \lambda_{2}\right), H^{0}\left(\lambda_{1}\right)$.

By lemma 5.2 any nontrivial cocycle of $Z^{2}\left(\mathfrak{g}, L\left((p-2) \lambda_{1}+\lambda_{2}\right)\right)$ must have a dominant weight of the form $p \lambda_{1}$. Therefore, cocycles of $Z^{2}\left(\mathfrak{g}, L\left((p-2) \lambda_{1}+\lambda_{2}\right)\right)$ with weights $p\left(2 \lambda_{1}+\lambda_{2}\right), 2 p \lambda_{2}$ are 0 . Thus $H^{0}\left(2 \lambda_{1}+\lambda_{2}\right), H^{0}\left(2 \lambda_{2}\right)$ can not be in ker $f$. We now prove that $H^{0}\left(\lambda_{1}\right)$ also can not be in ker $f$. To do this, we prove that cocycles with weight $p \lambda_{1}$ are coboundaries.

Let $\psi$ be some cocycle of weight $p \lambda_{1}$. Then

$$
\begin{gathered}
\psi\left(h_{1}, f_{1}\right)=x_{1} v_{0,0,0}, \psi\left(h_{2}, f_{1}\right)=x_{2} v_{0,0,0}, \psi\left(e_{2}, f_{3}\right)=x_{3} v_{0,0,0}, \\
\psi\left(h_{1}, f_{3}\right)=x_{4} v_{0,1,0}, \psi\left(h_{2}, f_{3}\right)=x_{5} v_{0,1,0,}, \\
\psi\left(f_{1}, f_{2}\right)=x_{6} v_{0,1,0}, \psi\left(f_{1}, f_{3}\right)=x_{7} v_{0,0,1},
\end{gathered}
$$

for some $x_{i} \in \mathcal{K}(i=1, \ldots, 7)$. Non-written components are zero.

From the cocyclicity conditions we obtain one cocycle of weight $p \lambda_{1}$ with the following non-zero components

$$
\psi\left(e_{2}, f_{3}\right)=v_{0,0,0}, \quad \psi\left(f_{1}, f_{2}\right)=v_{0,1,0}, \quad \psi\left(f_{1}, f_{3}\right)=v_{0,0,1} .
$$

It is easy to see that, $\psi$ is a coboundary $d \omega$, where $\omega\left(f_{1}\right)=v_{0,0,0}$. So, any cocycle of the weight $p \lambda_{1}$ is trivial. Thus $L\left(\lambda_{1}\right) \not \subset \operatorname{ker} f$. The case of the dual module $V=L\left(\lambda_{1}+(p-2) \lambda_{2}\right)$ may be treated in a similar way.

The case (b). According to proposition 4.2 the first cohomology groups are nonzero only for the following two modules $L\left((p-2) \lambda_{1}+2 \lambda_{2}\right), L\left(\lambda_{1}+(p-2) \lambda_{2}\right)$. By (5) we have

$$
\begin{gathered}
H^{3}\left(G_{1}, H^{0}\left((p-2) \lambda_{1}+2 \lambda_{2}\right)\right)^{(-1)} \cong H^{0}\left(2 \lambda_{1}\right) \oplus H^{0}\left(2 \lambda_{2}\right) \oplus H^{0}\left(\lambda_{1}+2 \lambda_{2}\right), \\
H^{3}\left(G_{1}, H^{0}\left(\lambda_{1}+(p-2) \lambda_{2}\right)\right)^{(-1)} \cong H^{0}\left(3 \lambda_{2}\right) \oplus H^{0}\left(\lambda_{1}+\lambda_{2}\right) .
\end{gathered}
$$

According to (24), (25), lemma 3.4, 5.3 and proposition 4.3 the exact sequence (8) can be rewritten as follows

$$
\begin{gathered}
0 \rightarrow L\left(\lambda_{1}\right) \rightarrow H^{3}\left(G_{1}, L\left((p-2) \lambda_{1}+2 \lambda_{2}\right)\right) \rightarrow \\
H^{0}\left(2 \lambda_{1}\right) \oplus H^{0}\left(2 \lambda_{2}\right) \oplus H^{0}\left(\lambda_{1}+2 \lambda_{2}\right) \rightarrow 0, \\
0 \rightarrow L\left(\lambda_{2}\right) \rightarrow H^{3}\left(G_{1}, L\left(\lambda_{1}+(p-2) \lambda_{2}\right)\right) \rightarrow \\
H^{0}\left(3 \lambda_{2}\right) \oplus H^{0}\left(\lambda_{1}+\lambda_{2}\right) \rightarrow 0 .
\end{gathered}
$$


By proposition 4.2 ,

$$
\begin{gathered}
H^{1}\left(\mathfrak{g}, L\left((p-2) \lambda_{1}+2 \lambda_{2}\right)\right) \otimes \mathfrak{g}^{*} \cong L\left(\lambda_{1}\right) \otimes \mathfrak{g} \cong \\
H^{0}\left(2 \lambda_{2}\right) \oplus H^{0}\left(\lambda_{1}+2 \lambda_{2}\right) \oplus H^{0}\left(\lambda_{1}\right), \\
H^{1}\left(\mathfrak{g}, L\left(\lambda_{1}+(p-2) \lambda_{2}\right)\right) \otimes \mathfrak{g}^{*} \cong L\left(\lambda_{2}\right) \otimes \mathfrak{g} \cong \\
H^{0}\left(3 \lambda_{2}\right) \oplus H^{0}\left(\lambda_{1}+\lambda_{2}\right) \oplus H^{0}\left(\lambda_{2}\right) .
\end{gathered}
$$

Let $V=L\left((p-2) \lambda_{1}+2 \lambda_{2}\right)$. From (26) and (28) we see that all composition factors $H^{0}\left(\lambda_{1}+2 \lambda_{2}\right), H^{0}\left(2 \lambda_{2}\right), H^{0}\left(\lambda_{1}\right)$ of the $G$-module $H^{1}\left(\mathfrak{g}, L\left((p-2) \lambda_{1}+2 \lambda_{2}\right)\right) \otimes \mathfrak{g}^{*}$ are in the module $H^{3}\left(G_{1}, L\left((p-2) \lambda_{1}+2 \lambda_{2}\right)\right)$. By lemma 5.2 cocycles have dominant weights equal to $p \lambda_{1}$. Therefore, $Z^{2}\left(\mathfrak{g}, L\left((p-2) \lambda_{1}+2 \lambda_{2}\right)\right)$ has no cocycles with weights $p\left(\lambda_{1}+2 \lambda_{2}\right), 2 p \lambda_{2}$. Therefore, $\operatorname{ker} f$ has no submodules isomorphic to $H^{0}\left(\lambda_{1}+\right.$ $\left.2 \lambda_{2}\right)$ or $H^{0}\left(2 \lambda_{2}\right)$. We now prove that $H^{0}\left(\lambda_{1}\right) \not \subset$ ker $f$. We prove that any cocycle $\psi$ with weight $p \lambda_{1}$ is a coboundary.

For any cocycle $\psi$ with weight $p \lambda_{1}$

$$
\begin{gathered}
\psi\left(h_{1}, f_{1}\right)=x_{1} v_{0,0,0,0}, \psi\left(h_{2}, f_{1}\right)=x_{2} v_{0,0,0,0}, \psi\left(e_{2}, f_{3}\right)=x_{3} v_{0,0,0,0}, \\
\psi\left(h_{1}, f_{3}\right)=x_{4} v_{1,0,0,0}, \psi\left(h_{2}, f_{3}\right)=x_{5} v_{1,0,0,0}, \psi\left(f_{1}, f_{2}\right)=x_{6} v_{1,0,0,0}, \\
\psi\left(e_{2}, f_{4}\right)=x_{7} v_{1,0,0,0}, \psi\left(h_{1}, f_{4}\right)=x_{8} v_{2,0,0,0}, \psi\left(h_{2}, f_{2}\right)=x_{9} v_{2,0,0,0}, \\
\psi\left(f_{2}, f_{3}\right)=x_{10} v_{2,0,0,0}, \psi\left(f_{1}, f_{3}\right)=x_{11} v_{0,1,0,0}, \\
\psi\left(f_{1}, f_{4}\right)=x_{12} v_{1,1,0,0}+x_{13} v_{0,0,0,1}, \psi\left(f_{3}, f_{4}\right)=x_{14} v_{1,0,0,1},
\end{gathered}
$$

for some $x_{i} \in \mathcal{K}(i=1, \ldots, 14)$. Non-written components are 0 .

From the cocyclicity conditions we obtain that such cocycles are linear combinations of the following two cocycles

$$
\begin{gathered}
\psi_{1}\left(e_{2}, f_{3}\right)=2 v_{0,0,0,0}, \quad \psi_{1}\left(f_{1}, f_{2}\right)=v_{1,0,0,0}, \\
\psi_{1}\left(f_{1}, f_{3}\right)=v_{0,1,0,0}, \psi_{1}\left(f_{1}, f_{4}\right)=v_{0,0,0,1} \\
\psi_{2}\left(e_{2}, f_{4}\right)=-v_{1,0,0,0}, \quad \psi_{2}\left(f_{2}, f_{3}\right)=-2 v_{2,0,0,0}, \\
\psi_{2}\left(f_{1}, f_{4}\right)=v_{0,0,0,1}, \psi_{2}\left(f_{3}, f_{4}\right)=v_{1,0,0,1} .
\end{gathered}
$$

Both cocycles are coboundaries: $\psi_{1}=d \omega_{1}$ and $\psi_{2}=d \omega_{2}$, where non-zero components of $\omega_{1}$ and $\omega_{2}$ are given by $\omega_{1}\left(f_{1}\right)=-v_{0,0,0,0}$ and $\omega_{2}\left(f_{4}\right)=-v_{2,0,0,0}$. So, any cocycle of weight $p \lambda_{1}$ is a coboundary. Therefore, $L\left(\lambda_{1}\right) \nsubseteq \operatorname{ker} f$.

Consider now the case $V=L\left(\lambda_{1}+(p-2) \lambda_{2}\right)$.

From (27) and (29) we see that $H^{1}\left(\mathfrak{g}, L\left(\lambda_{1}+(p-2) \lambda_{2}\right)\right) \otimes \mathfrak{g}^{*}$ and $H^{3}\left(G_{1}, L\left(\lambda_{1}+\right.\right.$ $\left.\left.(p-2) \lambda_{2}\right)\right)$ have equal composition factors $H^{0}\left(3 \lambda_{2}\right), H^{0}\left(\lambda_{2}+\lambda_{2}\right), H^{0}\left(\lambda_{2}\right)=L\left(\lambda_{2}\right)$. By lemma 5.2 a non-trivial 2-cocycle has dominant weight equal to $p \lambda_{2}$. Therefore, all cocycles of $Z^{2}\left(\mathfrak{g}, L\left(\lambda_{1}+(p-2) \lambda_{2}\right)\right)$, with $G$-weights $3 p \lambda_{2}, p\left(\lambda_{1}+\lambda_{2}\right)$ are trivial. Thus, the modules $H^{0}\left(3 \lambda_{2}\right), H^{0}\left(\lambda_{1}+\lambda_{2}\right)$ are not in $\operatorname{ker} f$. 
We now prove that $H^{0}\left(\lambda_{2}\right)$ is also not in $\operatorname{ker} f$. So, we need to prove that any 2-cocycle with weight $p \lambda_{2}$ is trivial.

Let $\psi$ be some cocycle of weight $p \lambda_{2}$. Then all components $\psi(X, Y)$ are zero except the following components

$$
\begin{gathered}
\psi\left(h_{1}, f_{2}\right)=x_{1} v_{0,0,0,0}, \psi\left(h_{2}, f_{2}\right)=x_{2} v_{0,0,0,0}, \psi\left(e_{1}, f_{3}\right)=x_{3} v_{0,0,0,0}, \\
\psi\left(e_{3}, f_{4}\right)=x_{4} v_{0,0,0,0}, \psi\left(h_{1}, f_{3}\right)=x_{5} v_{0,0,1,0}, \psi\left(h_{2}, f_{3}\right)=x_{6} v_{0,0,1,0}, \\
\psi\left(f_{1}, f_{2}\right)=x_{7} v_{0,0,0}, \psi\left(e_{2}, f_{4}\right)=x_{8} v_{0,0,1,0}, \\
\psi\left(h_{1}, f_{4}\right)=x_{9} v_{1,0,1,0}+x_{10} v_{0,1,0,0}, \\
\psi\left(h_{2}, f_{4}\right)=x_{12} v_{1,0,1,0}+x_{12} v_{0,1,0,0}, \psi\left(f_{2}, f_{3}\right)=x_{13} v_{1,0,1,0}+x_{14} v_{0,1,0,0}, \\
\psi\left(f_{2}, f_{4}\right)=x_{15} v_{1,1,0,0}+x_{16} v_{0,0,0,1}, \psi\left(f_{1}, f_{4}\right)=x_{17} v_{0,1,1,0}, \\
\psi\left(f_{3}, f_{4}\right)=x_{18} v_{1,1,1,0}+x_{19} v_{0,0,1,1}, \psi\left(e_{1}, f_{4}\right)=x_{20} v_{1,0,0,0},
\end{gathered}
$$

for some $x_{i} \in \mathcal{K}(i=1, \ldots, 20)$.

We have

$$
d \psi\left(e_{1}, f_{1}, f_{4}\right)=0 \Rightarrow x_{10}=0, x_{9}-x_{17}-x_{20}=0 .
$$

Then the equations $d \psi\left(h_{1}, e_{1}, f_{3}\right)=0, d \psi\left(h_{1}, e_{3}, f_{4}\right)=0$, $d \psi\left(h_{1}, f_{2}, f_{3}\right)=0$ gives us $x_{1}=x_{5}=x_{9}=0$.

Furthermore, from

$$
\begin{aligned}
& d \psi\left(f_{2}, f_{3}, f_{4}\right)=0, d \psi\left(e_{1}, f_{3}, f_{4}\right)=0, d \psi\left(f_{1}, f_{3}, f_{4}\right)=0, \\
& d \psi\left(e_{1}, f_{1}, f_{4}\right)=0, d \psi\left(e_{1}, f_{2}, f_{4}\right)=0, d \psi\left(e_{3}, f_{2}, f_{4}\right)=0
\end{aligned}
$$

we have $x_{4}=x_{16}=x_{14}=-x_{3}$ and $x_{18}=x_{13}=x_{15}=x_{20}=x_{17}=0$.

From $d \psi\left(e_{2}, f_{2}, f_{4}\right)=0, d \psi\left(e_{2}, f_{3}, f_{4}\right)=0, d \psi\left(e_{3}, f_{3}, f_{4}\right)=0$ we obtain $x_{8}=$ $x_{11}=x_{12}=x_{16}=x_{14}=x_{13}=0$. Finally, from $d \psi\left(e_{1}, f_{1}, f_{2}\right)=0, d \psi\left(e_{3}, f_{2}, f_{3}\right)=0$ we obtain $x_{7}=x_{2}=0$. So, $\psi=0$, if it has weight $p \lambda_{2}$. Thus $H^{0}\left(\lambda_{2}\right) \nsubseteq \mathbb{k e r} f$.

The case (c). By proposition 4.2 only the following two modules have non-zero first cohomology groups, $L\left((p-2) \lambda_{1}+\lambda_{2}\right)$ and $L\left(3 \lambda_{1}+(p-2) \lambda_{2}\right)$. We have proved above that $H^{2}\left(\mathfrak{g}, L\left(3 \lambda_{1}+(p-2) \lambda_{2}\right)\right) \cong L(0)^{(1)}$. Therefore, it is enough to check that ker $f=0$ for the module $L\left((p-2) \lambda_{1}+\lambda_{2}\right)$. According to (5),

$$
H^{3}\left(G_{1}, H^{0}\left((p-2) \lambda_{1}+\lambda_{2}\right)\right)^{(-1)} \cong H^{0}\left(\lambda_{2}\right) \oplus H^{0}\left(2 \lambda_{1}\right) \oplus H^{0}\left(\lambda_{1}+\lambda_{2}\right) .
$$

So, (30), lemma 3.5, 5.4 and proposition 4.3 and the exact sequence (8), gives us the following exact sequence

$$
\begin{gathered}
0 \rightarrow L\left(\lambda_{1}\right) \rightarrow H^{3}\left(G_{1}, L\left((p-2) \lambda_{1}+\lambda_{2}\right)\right) \rightarrow \\
H^{0}\left(\lambda_{2}\right) \oplus H^{0}\left(2 \lambda_{1}\right) \oplus H^{0}\left(\lambda_{1}+\lambda_{2}\right) \rightarrow 0 .
\end{gathered}
$$

By proposition 4.2 , we have

$$
H^{1}\left(\mathfrak{g}, L\left((p-2) \lambda_{1}+\lambda_{2}\right)\right) \otimes \mathfrak{g}^{*} \cong L\left(\lambda_{1}\right) \otimes \mathfrak{g} \cong
$$




$$
H^{0}\left(2 \lambda_{1}\right) \oplus H^{0}\left(\lambda_{1}+\lambda_{2}\right) \oplus H^{0}\left(\lambda_{1}\right) .
$$

By (31), (32) all composition factors of the $G$-module $H^{1}\left(\mathfrak{g}, L\left((p-2) \lambda_{1}+\lambda_{2}\right)\right) \otimes$ $\mathfrak{g}^{*}$ appear as a composition factors of $H^{3}\left(G_{1}, L\left((p-2) \lambda_{1}+\lambda_{2}\right)\right)$. If ker $f$ has such a composition factor, then $H^{2}\left(\mathfrak{g}, L\left((p-2) \lambda_{1}+\lambda_{2}\right)\right)$ has non-trivial cocycles with weights $2 p \lambda_{1}, p\left(\lambda_{1}+\lambda_{2}\right), p \lambda_{1}$. We will prove that this is impossible.

By lemma 5.2 the dominant weight of a non-trivial cocycle in $Z^{2}\left(\mathfrak{g}, L\left((p-2) \lambda_{1}+\lambda_{2}\right)\right)$ is $p \lambda_{1}$. Therefore, cocycles in $Z^{2}\left(\mathfrak{g}, L\left((p-2) \lambda_{1}+\lambda_{2}\right)\right)$ with weights $2 p \lambda_{1}, p\left(\lambda_{1}+\lambda_{2}\right)$ are 0 . we now prove that any cocycle $\psi$ with weight $p \lambda_{1}$ is 0 . All components $\psi(X, Y)$ of such a cocycle except the following components are 0 :

$$
\begin{aligned}
& \psi\left(e_{2}, f_{3}\right)=x_{1} v_{0,0,0,0,0,0}, \psi\left(e_{3}, f_{4}\right)=x_{2} v_{0,0,0,0,0,0}, \\
& \psi\left(e_{4}, f_{5}\right)=x_{3} v_{0,0,0,0,0,0}, \quad \psi\left(f_{1}, f_{2}\right)=x_{4} v_{0,1,0,0,0,0}, \\
& \psi\left(e_{1}, f_{4}\right)=x_{5} v_{0,1,0,0,0,0}, \psi\left(e_{4}, f_{6}\right)=x_{6} v_{0,1,0,0,0,0}, \\
& \psi\left(f_{1}, f_{3}\right)=x_{7} v_{1,1,0,0,0,0}+x_{8} v_{0,0,1,0,0,0}, \\
& \psi\left(e_{1}, f_{5}\right)=x_{9} v_{1,1,0,0,0,0}+x_{10} v_{0,0,1,0,0,0}, \\
& \psi\left(e_{3}, f_{6}\right)=x_{11} v_{1,1,0,0,0,0}+x_{12} v_{0,0,1,0,0,0}, \quad \psi\left(e_{2}, f_{4}\right)=x_{13} v_{1,0,0,0,0,0}, \\
& \psi\left(e_{3}, f_{5}\right)=x_{14} v_{1,0,0,0,0,0}, \psi\left(e_{2}, f_{5}\right)=x_{15} v_{2,0,0,0,0,0}, \\
& \psi\left(f_{2}, f_{4}\right)=x_{16} v_{0,1,1,0,0,0}, \quad \psi\left(f_{2}, f_{6}\right)=x_{17} v_{0,1,2,0,0,0}, \\
& \psi\left(e_{1}, f_{6}\right)=x_{33} v_{0,1,1,0,0,0}, \\
& \psi\left(f_{1}, f_{4}\right)=x_{18} v_{2,1,0,0,0,0}+x_{19} v_{1,0,1,0,0,0}+x_{20} v_{0,0,0,1,0,0}, \\
& \psi\left(e_{2}, f_{6}\right)=x_{21} v_{2,1,0,0,0,0}+x_{22} v_{1,0,1,0,0,0}+x_{23} v_{0,0,0,1,0,0} \\
& \psi\left(f_{2}, f_{5}\right)=x_{24} v_{1,1,1,0,0,0}+x_{25} v_{0,0,2,0,0,0}+x_{26} v_{0,1,0,1,0,0}, \\
& \psi\left(f_{3}, f_{4}\right)=x_{27} v_{1,1,1,0,0,0}+x_{28} v_{0,0,2,0,0,0}+x_{29} v_{0,1,0,1,0,0} \\
& \psi\left(f_{1}, f_{5}\right)=x_{30} v_{2,0,1,0,0,0}+x_{31} v_{1,0,0,1,0,0}+x_{32} v_{0,0,0,0,1,0}, \\
& \psi\left(f_{1}, f_{6}\right)=x_{34} v_{2,1,1,0,0,0}+x_{35} v_{1,0,2,0,0,0}+x_{36} v_{1,1,0,1,0,0}+ \\
& x_{37} v_{0,0,1,1,0,0}+x_{38} v_{0,1,0,0,1,0}+x_{39} v_{0,0,0,0,0,1}, \\
& \psi\left(f_{3}, f_{5}\right)=x_{40} v_{2,1,1,0,0,0}+x_{41} v_{1,0,2,0,0,0}+x_{42} v_{1,1,0,1,0,0}+ \\
& x_{43} v_{0,0,1,1,0,0}+x_{44} v_{0,1,0,0,1,0}+x_{45} v_{0,0,0,0,0,1}, \\
& \psi\left(f_{3}, f_{6}\right)=x_{46} v_{1,1,2,0,0,0}+x_{47} v_{0,0,3,0,0,0}+x_{48} v_{0,1,1,1,0,0}+x_{49} v_{0,1,0,0,0,1}, \\
& \psi\left(f_{4}, f_{5}\right)=x_{50} v_{3,1,1,0,0,0}+x_{51} v_{2,0,2,0,0,0}+x_{52} v_{2,1,0,1,0,0}+
\end{aligned}
$$




$$
\begin{gathered}
x_{53} v_{0,0,0,2,0,0}+x_{54} v_{1,0,1,1,0,0}+x_{55} v_{0,0,1,0,1,0}, \\
\psi\left(f_{4}, f_{6}\right)=x_{56} v_{2,1,2,0,0,0}+x_{57} v_{1,0,3,0,0,0}+x_{58} v_{0,1,0,2,0,0}+x_{59} v_{1,1,1,1,0,0}+ \\
x_{60} v_{0,0,2,1,0,0}+x_{61} v_{1,1,0,0,0,1}+x_{62} v_{0,0,1,0,0,1}++x_{63} v_{0,1,1,0,1,0}, \\
\psi\left(f_{5}, f_{6}\right)=x_{64} v_{3,1,2,0,0,0}+x_{65} v_{2,0,3,0,0,0}+x_{66} v_{1,1,0,2,0,0}+x_{67} v_{2,1,1,1,0,0}+ \\
x_{68} v_{1,0,2,1,0,0}+x_{69} v_{0,0,1,2,0,0}+x_{70} v_{1,1,1,0,1,0}+x_{71} v_{0,0,2,0,1,0}+ \\
x_{72} v_{0,1,0,1,1,0}+x_{73} v_{2,1,0,0,0,1}+x_{74} v_{1,0,1,0,0,1}+x_{75} v_{0,0,0,1,0,1},
\end{gathered}
$$

where $x_{i} \in \mathcal{K}(i=1, \ldots, 75)$.

From $d \psi\left(h_{i}, f_{1}, f_{6}\right)=0, d \psi\left(h_{i}, f_{1}, f_{5}\right)=0, d \psi\left(h_{i}, e_{2}, f_{3}\right)=0, i=1,2$, it follows that

$$
\psi\left(h_{i}, f_{1}\right)=\psi\left(h_{i}, f_{3}\right)=\psi\left(h_{i}, f_{4}\right)=\psi\left(h_{i}, f_{5}\right)=\psi\left(h_{i}, f_{6}\right)=0, i=1,2
$$

From

$$
\begin{gathered}
d \psi\left(f_{4}, f_{5}, f_{6}\right)=0, d \psi\left(f_{3}, f_{5}, f_{6}\right)=0, d \psi\left(f_{2}, f_{5}, f_{6}\right)=0, \\
d \psi\left(f_{3}, f_{4}, f_{6}\right)=0, d \psi\left(f_{3}, f_{4}, f_{5}\right)=0, d \psi\left(f_{1}, f_{2}, f_{6}\right)=0, \\
d \psi\left(f, f_{2}, f_{5}\right)=0, d \psi\left(e_{2}, f, f_{6}\right)=0, d \psi\left(e_{4}, f_{5}, f_{6}\right)=0,
\end{gathered}
$$

it follows that the following components $\psi\left(f_{5}, f_{6}\right), \psi\left(f_{3}, f_{6}\right), \psi\left(f_{3}, f_{5}\right), \psi\left(f_{2}, f_{6}\right)$, $\psi\left(f_{2}, f_{5}\right), \psi\left(f_{1}, f_{6}\right), \psi\left(f_{1}, f_{5}\right), \psi\left(f_{1}, f_{2}\right), \psi\left(e_{4}, f_{6}\right)$, $\psi\left(e_{4}, f_{5}\right), \psi\left(e_{2}, f_{6}\right)$ are zero and $\psi\left(f_{4}, f_{6}\right)=x_{62} v_{0,0,1,0,0,1}$, $\psi\left(f_{4}, f_{5}\right)=x_{62} v_{0,0,1,0,1,0}, \psi\left(f_{3}, f_{4}\right)=-2 x_{62} v_{0,0,2,0,0,0}$.

From the conditions

$$
\begin{array}{r}
d \psi\left(e_{4}, f_{3}, f_{4}\right)=0, d \psi\left(e_{3}, f_{4}, f_{6}\right)=0, d \psi\left(e_{1}, f_{4}, f_{5}\right)=0, \\
d \psi\left(e_{1}, f_{4}, f_{6}\right)=0
\end{array}
$$

we see that the following components

$$
\psi\left(e_{1}, f_{4}\right), \psi\left(f_{4}, f_{6}\right), \psi\left(f_{1}, f_{6}\right), \psi\left(f_{3}, f_{4}\right), \psi\left(e_{1}, f_{5}\right), \psi\left(f_{2}, f_{4}\right), \psi\left(e_{3}, f_{6}\right)
$$

are zero. Finally, from the equations

$$
\begin{aligned}
& d \psi\left(f_{1}, f_{2}, f_{4}\right)=0, d \psi\left(e_{3}, f_{2}, f_{4}\right)=0, d \psi\left(e_{1}, e_{2}, f_{4}\right)=0, \\
& d \psi\left(e_{1}, e_{3}, f_{5}\right)=0, d \psi\left(e_{6}, f_{3}, f_{5}\right)=0, d \psi\left(e_{3}, f_{3}, f_{4}\right)=0
\end{aligned}
$$

we have

$$
\begin{array}{r}
\psi\left(f_{1}, f_{4}\right)=\psi\left(e_{2}, f_{4}\right)=\psi\left(e_{3}, f_{5}\right)=\psi\left(f_{1}, f_{3}\right)=\psi\left(e_{3}, f_{4}\right)= \\
\psi\left(e_{2}, f_{3}\right)=0 .
\end{array}
$$

Therefore, any cocycle in $Z^{2}\left(\mathfrak{g}, L\left((p-2) \lambda_{1}+\lambda_{2}\right)\right)$ with weight $p \lambda_{1}$ is zero. Thus ker $f=0$.

Theorem 1.1 is proved completely. 
Acknowledgements. The authors are grateful to prof. C. Löfwall for attention to our results and INTAS foundation and the Royal Swedish Academy of Sciences for support.

\section{References}

[1] H.H. Andersen, The strong linkage principle, J. Reine Angew. Math., 315 (1980), 53-59.

[2] H.H. Andersen, J.C. Jantzen, Cohomology of induced representations for algebraic groups, Math. Ann., 269 (1984), 487-525.

[3] N. Bourbaki, Groupes et algèbres de Lie, Chap. 2 et 3, Paris: Hermann, 1972.

[4] B. Braden, Restricted representations of classical Lie algebras of types $A_{2}$ and $B_{2}$, Bull. Amer. Math. Soc., 73 (1967), 482-485.

[5] Chiu Sen, Cohomology of graded Lie algebras of Cartan type $S(n, m)$, Chin. Ann. Math., 10B(1), 1989, 105-114.

[6] A.S. Dzhumadil'daev, On the defomations of classical Lie algebras, Uspechi Mat. Nauk, 31(1976), No.3, 211-212.

[7] A.S. Dzhumadil'daev, On the cohomology of modular Lie algebras, 119 (161), 1982, n.9, 132-149.

[8] A.S. Dzhumadil'daev, Cohomology and nonsplit extensions of modular Lie algebras, Contemp.Math. 131(1992), part 2, p.31-43.

[9] G. Hochschild, Cohomology of restricted Lie algebras, Amer. J. Math., 76 (1954),555-580.

[10] J.E. Humphreys, Modular representations of classical Lie algebras and semisimple groups, J. Algebra, 19 (1971), 51-79.

[11] J.C. Jantzen, Representations of algebraic groups, Pure and Applied Mathematics, 131, Boston,1987.

[12] J.C. Jantzen, First cohomology groups for classical Lie algebras, Progress in mathematics, 95 (1991), 289-315.

[13] A.I. Kostrikin, Parametrical family of simple Lie algebras, Izv. AN SSSR, Ser. mat., 34 (1970), 744-756.

[14] L.M. Reed, A positive characteristic resolution problem for $S L(3, k)$, J. Algebra, 198 (1997), 57-100.

[15] A.N. Rudakov, Dimensions of some representations of semisimple Lie algebras of classical types over a field of finite characteristic , Trudy Semin. Petrovskogo, No. 3, 1978, 147-160.

[16] J.B. Sullivan, Lie algebra cohomology at irreducible modules, J. Math., 23 (1979), 363-373. 
[17] J.B. Sullivan, The second Lie algebra cohomology group and Weyle modules, J. Math., 86 (1980), 321-326.

[18] F.D. Veldkamp, The center of the universal enveloping algebra of a Lie algebra in characteristic p, Ann. Scient. Norm. Sup., 5, 1972, 217-240.

This article may be accessed via WWW at http://www.rmi.acnet.ge/hha/ or by anonymous ftp at

ftp://ftp.rmi.acnet.ge/pub/hha/volumes/2002/n2a6/v4n2a6.(dvi,ps,pdf)

A.S. Dzhumadil'daev askar@math.kz

S. Demirel University,

Toraygirov 19, Almaty,

Kazakhstan

S.S. Ibraev

Institute of Mathematics,

Almaty,

Kazakhstan 